\title{
MULTISYMPLECTIC VARIATIONAL INTEGRATORS FOR NONSMOOTH LAGRANGIAN CONTINUUM MECHANICS
}

\author{
FRANÇOIS DEMOURES ${ }^{1,2}$, FRANÇOIS GAY-BALMAZ ${ }^{2}$ and \\ TUDOR S. RATIU ${ }^{3,4}$ \\ ${ }^{1}$ Department of Mathematics, Imperial College, London, UK; \\ email: demoures@lmd.ens.fr \\ ${ }^{2}$ LMD/IPSL, CNRS, École Normale Supérieure, PSL Research University, \\ Université Paris-Saclay, Sorbonne Universités, Paris, France; \\ email: francois.gay-balmaz@lmd.ens.fr \\ ${ }^{3}$ Department of Mathematics, Shanghai Jiao Tong University, 800 Dongchuan Road, \\ Minhang District, 200240 Shanghai, China \\ ${ }^{4}$ Section de Mathématiques, École Polytechnique Fédérale de Lausanne, \\ CH 1015 Lausanne, Switzerland; \\ email: tudor.ratiu@epfl.ch
}

Received 13 August 2015; accepted 2 June 2016

\begin{abstract}
This paper develops the theory of multisymplectic variational integrators for nonsmooth continuum mechanics with constraints. Typical problems are the impact of an elastic body on a rigid plate or the collision of two elastic bodies. The integrators are obtained by combining, at the continuous and discrete levels, the variational multisymplectic formulation of nonsmooth continuum mechanics with the generalized Lagrange multiplier approach for optimization problems with nonsmooth constraints. These integrators verify a spacetime multisymplectic formula that generalizes the symplectic property of time integrators. In addition, they preserve the energy during the impact. In the presence of symmetry, a discrete version of the Noether theorem is verified. All these properties are inherited from the variational character of the integrator. Numerical illustrations are presented.
\end{abstract}

2010 Mathematics Subject Classification: 65D99, 49M30, 74B20, 74M20 (primary); 49J52, 53D20, 35Q74 (secondary) 


\section{Introduction}

Numerical methods addressing contact problems are in high demand in a multitude of multibody dynamics applications. As opposed to formulations of models in smooth mechanics, contact problems must deal with the singularities separating two regions of a continuous medium, such as the contact set of colliding elastic bodies. Modeling these singularities and numerically handling them, in a way that respects the crucial inequality conditions and conserved quantities expected from the dynamics, has been an important scientific challenge. No complete satisfactory solutions meeting both physical and computational constraints exist to this day. The results of this paper contribute to the solution of this long-standing problem. Our approach combines methods from nonsmooth optimization and multisymplectic field theory.

A classical point of view in physics consists in deriving the equations of motion from variational principles, or, equivalently, computing the critical points of an action functional. In the presence of constraints, such as impenetrability, propagation of singularities in an elastic body, solid-fluid boundaries, such a critical point condition is not sufficient to derive the dynamics. One needs to consider an optimization problem for the action functional, subject to conditions appropriately derived from such type of constraints. This leads to the setting of variational analysis which provides powerful tools such as the generalized Lagrange multiplier theorem, normal cone analysis, Kuhn-Tucker conditions, and, more generally, nonsmooth analysis. Developing the discrete analogue of these methods allows us to start the development of structure preserving numerical schemes for nonsmooth continuum mechanics with constraints, using variational discretization of multisymplectic field theories with constraints.

Description of the paper. We combine the aforementioned variational multisymplectic approaches with the generalized Lagrange multiplier approach for constrained optimization problems, as formulated, for example, in Clarke [18], Rockafellar and Wets [120], Dontchev and Rockafellar [31], in order to develop the theory of multisymplectic variational discretization for nonsmooth continuum mechanics with constraints. The main steps underlying our approach are the following.

First, we formulate the smooth unconstrained problem as a multisymplectic Lagrangian field theory, by identifying the space of fields, the Lagrangian density, the spacetime Hamilton principle, and the associated Cartan forms. Second, we extend this multisymplectic Lagrangian formulation to a nonsmooth unconstrained setting, following Fetecau et al. [41], identifying the types of singularities arising in the problem. This is done by using an extension of the 
spacetime Hamilton principle that allows for the treatment of certain types of singularities in the fields and automatically produces the needed jump conditions at the singularities. This, in turn, is accomplished by considering both vertical and horizontal field variations in the variational principle, as explained in the body of the paper. Third, we include constraints in this formulation, appealing to the generalized Lagrange multiplier approach in Rockafellar and Wets [120] for nonsmooth optimization, through inequality constraints. This approach produces a necessary condition on the critical points of a functional restricted to a constrained subset, and is based on the concept of the normal cone. In our context, we formally apply this theory to the Lagrangian action functional of the problem, in the extended nonsmooth setting presented in Fetecau et al. [41], and relative to both vertical and horizontal variations. We show that the critical fields still verify a generalized multisymplectic form formula, as in the smooth unconstrained case, which is the spacetime extension of the symplectic property of the solution flow in classical mechanics. In addition, in presence of symmetries, a Noether theorem is still available, exactly as in the smooth unconstrained case. Fourth, we discretize in spacetime this smooth constrained variational theory. Although, as is apparent from our development, a general treatment is possible, we work, for simplicity, on a $1+1$-dimensional spacetime, with a discretization based on a rectangular mesh. As in the continuous case, the constraint is introduced directly at the level of the variational formulation for the discrete action functional, using the generalized Lagrange multiplier approach. Both vertical and horizontal variations are considered. The choice of the allowed horizontal variations becomes crucial in the discrete case, since it requires a spacetime grid adaptation, and the enforcement of stationarity under these horizontal variations yields discrete energy balance or discrete balance of configurational forces. In our case, the horizontal variations are chosen to be associated to the time of impact, and therefore result in the conservation of the discrete global energy during the impact. Applying the generalized Lagrange multiplier approach with respect to the constraint in the discrete setting, relative to both vertical and horizontal variations, yields the desired numerical scheme. We show that this scheme is multisymplectic in the sense that it verifies a generalized discrete multisymplectic formula with constraints. In the presence of symmetry, we show that a discrete version of the Noether theorem is verified. Both these properties are inherited from the discrete spacetime variational character of the integrator. Fifth, we present two concrete examples.

In absence of constraints, our schemes recover the multisymplectic integrators derived in Marsden et al. [82]. In the particular case of classical mechanics, that is, when spacetime reduces to time, our integrator recovers the collision variational integrators of Fetecau et al. [42]. 


\section{Summary of the main results}

- Development of the variational multisymplectic formulation of nonsmooth mechanics with constraints (Theorem 3).

- Derivation of the Noether Theorem and the multisymplectic form formula in the nonsmooth setting with constraints (Section 3.2.2 and (35)).

- Discretization of the geometric setting on $1+1$-dimensional spacetime (Section 4.1).

- Derivation of the multisymplectic numerical schemes for two specific cases (Theorems 5 and 7).

- Derivation of the discrete Noether Theorem (Theorem 6, Section 4.3.6) and multisymplectic form formula ((70), Section 4.3.5).

- Illustration with the examples of the collisions of a geometrically exact elastic beam on a plate and the collision of two elastic bars (Section 5).

- Extension of discrete Lie group integrators to rectangular spacetime discretizations (Section 5.1).

Nonsmooth mechanics has a long history. We believe that incorporating its foundations within the geometric mechanics framework is not only rewarding from the modeling point of view, but it should also lead to new computational approaches to contact problems. Given that current numerical methods still cannot satisfactorily deal with the difficult issues inherent to nonsmooth mechanics, our drastically different approach can offer new insights.

Concise historical overview. We close this introduction by giving a brief historical synopsis of the methods that have been developed in the area of nonsmooth mechanics and its discretization. Since this time-honored research topic is extensive and involves many methods and points of view, it is impossible to do justice to all who contributed to the development of this important subject and so we give here only the highlights. Excellent reviews of some of the topics are found in Prékopa [107] and Curnier [25], from which we cite, with the permission of the author. 'The impenetrability and the intensility conditions (i.e., the contact force is a pressure, not a tension) were thoroughly discussed by Bernoulli, Johann (1710) (Johann Bernoulli was the first who formulated the principle of virtual work, according to Lagrange [74, volume 1, pages 21-22]; it appears as an axiom of theoretical mechanics on page 27.), Euler [37], and Fourier [49]. The impenetrability condition $(g \geqslant 0)$ (i.e., the reaction force has to vanish as soon as the point in contact lifts off) was first formulated by Cournot 
[24] and the intensility condition $(p \leqslant 0)$ by Ostrogradsky [99]'. (That is, the reaction force has to vanish as soon as the point in contact lifts off.) These two conditions were effectively combined by Hertz [64] and Signorini [122], together with the gap-contact alternative: either $g>0$ and $p=0$, or $g=0$ and $p \leqslant 0$ if there is contact. The complementarity condition $g \cdot p=0$, which enforces this alternative, was explicitly added by Moreau [86], probably inspired by the introduction of such a condition by Kuhn and Tucker [73] in optimization (independently of the thesis of Karush [71], which remained unnoticed until Kuhn and Tucker [73]). Therefore, although the three unilateral contact conditions are usually called Signorini's conditions, it seems fair to attribute them to Hertz, Signorini and Moreau in trio. The term unilateral was coined by Clausius [19] and spread out by Duhem [32], Painlevé [101] and Delassus [27]. It is no surprise if many contact mechanicians became famous thermomechanicians, in view of the role played by inequalities in both fields. Although they were clearly stated by de Lagrange [74], the conditions that the reaction is arbitrary and its work zero in a bilateral contact were (and still are) never explicitly written down because the former is rather trivial and the latter redundant in this case. This is perhaps why, in case of unilateral contact, it took a long time before Moreau [86] added the complementarity condition to the unilateral inequality conditions already formulated by 1834 '. The complementarity condition states that the work of the reaction force is zero in a bilateral or unilateral contact.

The general point of view, adopted to describe the motion of a body with unilateral contact, has been largely based on the work of Moreau [85, 8789] and Rockafellar $[108,109,111]$ in nonsmooth convex analysis, who were probably influenced by the lecture notes of Fenchel [40]. The unilateral contact requirement combines a geometric condition of impenetrability, a static condition of inattraction, and an energetic condition of complementarity (expressing that the work developed by the constraining force through the constrained displacement must vanish). See also Clarke [18] for a systematic development of this approach.

In Moreau [90] and Rockafellar [110], it was shown that the solutions $x$ to the problem of minimizing a proper convex function $f$ over a nonempty convex set $C$ can, alternatively, be described as the points $x$ satisfying $0 \in$ $\partial f(x)+\partial I_{C}(x)$, where $I_{C}$ is the indicator function of the convex set $C$ and $\partial f, \partial I_{C}$ denote the subgradients. In Rockafellar [112], optimization problems for integral functionals that are convex functionals were studied. These results were for the first time applied to nonsmooth mechanics in Moreau [90-92]; see also Moreau [93, 94, 96] and Moreau and Panagiotopoulos [98]. Particularly important results for nonsmooth mechanics were developed in Moreau [92]. For example, for unilateral contact without friction, the subgradient of $I_{C}$ was used to express the normal reaction, directed towards the permitted region. For static problems, a 
Lagrangian was considered in order to define the equilibrium forces at the time of contact.

In order to deal with impulses at contact points, differential inclusion was first used for contact mechanics in Filippov [44-46]. Combining differential inclusions with convex analysis, a formulation of unilateral contact between rigid bodies was derived in Moreau [94, 95]. A formulation of the energy potential for a distributed unilateral contact, associated to the inequality constraints expressing impenetrability properties, was proposed in He et al. [62]. The case of unilateral contact with friction was also investigated in, for example, Papadopoulos [103] and Moreau [95, 97].

After these above mentioned theoretical developments, the main research was then concentrated towards the development of numerical methods to solve optimization problems under inequality constraints, for example, in Hughes et al. [66]. Examples of such methods are the condensation method, the interior method, the barrier method, the penalty method, the exact proximity method, the exact multiplier method, and the augmented Lagrangian multiplier method. As pointed out in Curnier [26], 'the (primal) approximate penalty method was discovered for equality constraints (Euler [36], Carnot [16], Cournot [23], Thomson and Tait [127]) and analyzed (Courant [20-22], Beltrami [8], Fiacco and McCormick [43]) much before it was extended to inequality constraints (Rockafellar [113], Bertsekas [9-11], Auslender (1986)).

The (primal) exact proximity method for inequality constraints or nonsmooth optimization (a smart transformation of the approximate penalty method) was discovered by Moreau [88] at the nonsmooth convex functional level and also by Yosida [132] at the multivalued monotone operator level. Hence it is also known as the Moreau and Yosida regularization. It was implemented by Martinet [84], analyzed by Rockafellar [115] and generalized in Attouch and Wets [5-7] and Rockafellar and Wets [120]. To my knowledge, it was applied to a unilateral contact problem for the first time in a preedition (2006) of this book. (This refers to Curnier [26].)

The (mixed) exact multiplier method was also invented for equality constraints (Euler [35], Lagrange [74, 75], Legendre [77]) quite before it was adapted to inequality constraints (Fourier [49], Gauß [51], Ostrogradsky [99, 100], Painlevé [101], Farkas [39], Bouligand [13], Karush [71], Kuhn and Tucker [73], Moreau [85, 89], Ekeland and Temam [33], Hughes et al. [66], Glowinski et al. [56], Klarbring [72] ... )'.

To our knowledge, the first solution to a nonlinear optimization problem with inequality constraints was carried out by Fourier [49] who also set the bases for the parametric solutions of homogeneous linear inequalities in Fourier [50]. The first rigorous proof of this fundamental theorem about homogeneous linear 
inequalities is due, as far as we know, to Farkas [38]. We refer to Prékopa [107] for a thorough discussion and comparison of the contributions of Fourier and Farkas to this subject and the presentation of the Fourier inequality principle by Hamel [60], [61, pages 69-70 and 517-518].

According to Curnier [26]: 'Finally, the recent (mixed) exact augmented Lagrangian multiplier method was again introduced for equality constraints (Hestenes [65], Powell [105], Haarhoff and Buys [58], beside a hint in Arrow and Solow [4] ...), slightly before it was extended to inequality constraints (Wierzbicki [129], Buys [15], Bertsekas [12], Glowinski and Marocco [54], Rockafellar [114], Fortin and Glowinski [48], Glowinski and Le Tallec [55], Ito and Kunisch [68]) and to unsymmetric problems without functional (Alart and Curnier [2] ...). It was recognized as the proximity method applied to the dual part of the classical Lagrange multiplier method in Rockafellar [116-118] in convex analysis and in Alart [1], Hefgaard and Curnier [63], Pietrzak [106], Glocker [52], Leine and Glocker [78], Leine and Nijmeijer [79]'. The work of Papadopoulos and Taylor $[104,126]$ was influenced by the augmented Lagrangian formulation. Moreover, a penalty Lagrangian method was proposed in Taylor and Papadopoulos [126].

The barrier method was introduced in contact problems within the framework of finite element methods in, for example, Kane et al. [70]. In a variational Lagrangian setting, this technique is based on the variational principle

$$
\delta \int_{0}^{T}\left[L(q, \dot{q})-I_{C}(q)\right] d t=0 .
$$

For the theoretical point of view, associated to this method, see Forsgren et al. [47].

We refer to Armero and Petocz [3], Laursen [76], Pandolfi et al. [102], Wriggers [130], Cirak and West [17], Wriggers and Laursen [131], Studer et al. [125], Johnson et al. [69], and references therein for more recent work in computational contact mechanics.

\section{Variational multisymplectic geometry}

In this section, we recall several concepts from smooth and nonsmooth variational multisymplectic geometry that are crucial for the development of our approach.

In Section 2.1, we review the basic geometric objects needed to formulate a Lagrangian field theory, using the formulation, notations, conventions, and definitions in Gotay et al. [57]. We also recall the standard Hamilton variational principle leading to the covariant Euler-Lagrange equations. In Section 2.2, we 
review, from Marsden et al. [82], an extension of this variational principle in which variations of the field with respect to the spacetime parametrizations are also considered, in addition to the usual fiber variations. This setting turns out to be a natural variational framework to introduce the Cartan forms and to derive the covariant Noether theorem and the multisymplectic form formula. In Section 2.3, we review, from Fetecau et al. [41], how the extended variational setting naturally provides an appropriate framework for the variational multisymplectic formulation of nonsmooth continuum mechanics. We end this section by formulating, in Section 2.4, an extension of the Noether theorem and the multisymplectic form formula to the nonsmooth setting.

2.1. Lagrangian field theoretical framework. Let $X$ be an oriented manifold with piecewise smooth boundary, the spacetime, and let $\pi_{X Y}: Y \rightarrow X$ be a finitedimensional fiber bundle, the configuration bundle, the field theoretic analogue of the configuration space of classical mechanics. The physical fields $\varphi: X \rightarrow Y$ are sections of this bundle, that is, $\pi_{X Y} \circ \varphi=\mathrm{id}_{X}\left(\operatorname{id}_{X}=\right.$ identity map on $\left.X\right)$.

The field theoretical analogue of the tangent bundle of classical mechanics is played by the first jet bundle $\pi_{Y J^{1} Y}: J^{1} Y \rightarrow Y$; it is an affine bundle over $Y$, whose fiber at $y \in Y_{x}:=\pi_{X Y}^{-1}(x)$ consists of linear maps $\gamma: T_{x} X \rightarrow T_{y} Y$ satisfying $T \pi_{X Y} \circ \gamma=\mathrm{id}_{T_{X} X}$. Note that $J^{1} Y$ can also be regarded as the total space of a bundle over $X$, namely, $\pi_{X J^{1} Y}:=\pi_{X Y} \circ \pi_{Y J^{1} Y}: J^{1} Y \rightarrow X$. The derivative of a field $\varphi$ can be regarded as a section of $\pi_{X J^{1} Y}$, by writing $x \in X \mapsto j^{1} \varphi(x):=$ $T_{x} \varphi \in J_{\varphi(x)}^{1} Y$. The section $j^{1} \varphi$ is called the first jet extension of $\varphi$.

We let $\operatorname{dim} X=n+1$ and the fiber dimension of $Y$ be $N$. The coordinates on $X$ are denoted $x^{\mu}, \mu=0,1,2, \ldots, n$, the fiber coordinates on $Y$ are denoted $y^{A}, A=1, \ldots, N$, and the induced coordinates on the fiber of $J^{1} Y \rightarrow Y$ are denoted $v_{\mu}^{A}$. In such coordinates, the first jet extension reads $x^{\mu} \mapsto\left(x^{\mu}, \varphi^{A}\left(x^{\mu}\right)\right.$, $\left.\varphi^{A}{ }_{, v}\left(x^{\mu}\right)\right)$.

A Lagrangian density $\mathcal{L}: J^{1} Y \rightarrow \Lambda^{n+1} X$ is a smooth bundle map over $X$. In coordinates, we write $\mathcal{L}(\gamma)=L\left(x^{\mu}, y^{A}, v^{A}{ }_{\mu}\right) d^{n+1} x$. The associated action functional is $S(\varphi):=\int_{X} \mathcal{L}\left(j^{1} \varphi(x)\right)$. Consider a variation of $\varphi$ provided by a curve $\varphi^{\varepsilon}=\eta^{\varepsilon} \circ \varphi$, where $\eta^{\varepsilon}$ is the flow of a vertical vector field on $Y$ which is compactly supported in $X$. A section is critical for such variations of $S$ if and only if it verifies the covariant Euler-Lagrange equations, given in coordinates by

$$
\frac{\partial L}{\partial \varphi^{A}}-\partial_{\mu} \frac{\partial L}{\partial \varphi^{A}, \mu}=0, \quad \mu=0,1, \ldots, n .
$$

We recall below an extension of this variational principle, where horizontal variations are also considered. 


\subsection{Extended variational framework, multisymplectic form formula, and} Noether theorem. We now recall, from Marsden et al. [82], an extended variational approach to multisymplectic field theory, in which arbitrary, and not only vertical, variations of sections are considered. This extension is motivated by the following facts. First, it allows a complete variational characterization of the Cartan form. Second, it is the appropriate setting for the derivation of the multisymplectic form formula which extends the symplectic property of flows from mechanics to field theory. Finally, horizontal variations allow a rigorous derivation of the jump conditions when the fields are allowed to have singularities (see Fetecau et al. [41]).

In addition to the configuration bundle $\pi_{X Y}: Y \rightarrow X$, we fix a manifold $U$ with piecewise smooth boundary and with $\operatorname{dim} U=\operatorname{dim} X$. In the extended variational framework, a configuration is a smooth map $\phi: U \rightarrow Y$, such that $\phi_{X}:=\pi_{X Y} \circ \phi: U \rightarrow X$ is an embedding. The corresponding physical field $\varphi:=\phi \circ \phi_{X}^{-1}: \phi_{X}(U) \rightarrow Y$ is a section of the configuration bundle $\pi_{X Y}: Y \rightarrow X$ restricted to $U_{X}:=\phi_{X}(U) \subset X$. The space of all configurations is thus the set of smooth maps $\mathcal{C}(Y)=\left\{\varphi: U \rightarrow Y \mid \pi_{X Y} \circ \phi: U \rightarrow X\right.$ is an embedding $\}$. In this context, the action functional associated to a given Lagrangian density $\mathcal{L}$ is $\mathfrak{S}(\phi)=\int_{U_{X}} \mathcal{L}\left(j^{1}\left(\phi \circ \phi_{X}^{-1}\right)\right)$. A variation of $\phi$ is given by a family of maps $\phi^{\varepsilon}=\eta^{\varepsilon} \circ \phi$, where $\eta^{\varepsilon}$ are $\pi_{X Y}$-bundle automorphisms with $\eta^{0}=\mathrm{id}_{Y}$. Defining the vector field $V:=d /\left.d \varepsilon\right|_{\varepsilon=0} \eta^{\varepsilon}$, we have

$$
\left.\frac{d}{d \varepsilon}\right|_{\varepsilon=0} \mathfrak{S}\left(\eta^{\varepsilon} \circ \phi\right)=-\int_{U_{X}}\left(j^{1} \varphi\right)^{*} \mathbf{i}_{j^{1} V} \Omega_{\mathcal{L}}+\int_{\partial U_{X}}\left(j^{1} \varphi\right)^{*} \mathbf{i}_{j^{1} V} \Theta_{\mathcal{L}}
$$

(see Marsden et al. [82]), where $\Theta_{\mathcal{L}}$ is the Cartan $(n+1)$-form on $J^{1} Y$ and $\Omega_{\mathcal{L}}:=$ $-\mathbf{d} \Theta_{\mathcal{L}}$. In local coordinates,

$$
\begin{aligned}
& \Theta_{\mathcal{L}}=\frac{\partial L}{\partial \varphi^{A}{ }_{, \mu}} d y^{A} \wedge d^{n} x_{\mu}+\left(L-\frac{\partial L}{\partial \varphi^{A}{ }_{, \mu}} \varphi^{A}{ }_{, \mu}\right) d^{n+1} x \\
& \Omega_{\mathcal{L}}=d y^{A} \wedge \mathbf{d}\left(\frac{\partial L}{\partial \varphi^{A}{ }_{, \mu}}\right) \wedge d^{n} x_{\mu}+\mathbf{d}\left(\frac{\partial L}{\partial \varphi^{A}{ }_{, \mu}} \varphi^{A}{ }_{, \mu}-L\right) \wedge d^{n+1} x,
\end{aligned}
$$

where $d^{n} x_{\mu}:=\mathbf{i}_{\partial_{\mu}} d^{n+1} x$. In (2), $j^{1} V$ denotes the first jet extension of $V$, defined as the vector field on $J^{1} Y$ whose flow is the first jet extension of the flow of $V$. Note that the forms $\Theta_{\mathcal{L}}$ and $\Omega_{\mathcal{L}}$ can be completely characterized with the help of the variational formula (2). We give the local expression of the terms in (2) later in the nonsmooth case. For the moment, we just mention that stationarity of the action relative to vertical variations yields the covariant Euler-Lagrange equations (1) and stationarity relative to horizontal variations yields the balance 
of energy and the balance of configurational forces

$$
\frac{d}{d x^{\mu}}\left(\frac{\partial L}{\partial \varphi^{A}, \mu} \varphi_{, \nu}^{A}-L \delta_{v}^{\mu}\right)+\frac{\partial L}{\partial x^{v}}=0, \quad v=0,1, \ldots, n .
$$

The multisymplectic form formula follows from (2) (see Marsden et al. [82, Theorem 4.1]). Let $\phi$ be a solution of the covariant Euler-Lagrange equations and $V, W$ solutions of the first variation of the Euler-Lagrange equations at $\phi$. Then $V, W, \phi$ verify the multisymplectic form formula:

$$
\int_{\partial \phi_{X}\left(U^{\prime}\right)}\left(j^{1} \varphi\right)^{*} \mathbf{i}_{j^{1} V} \mathbf{i}_{j^{1} W} \Omega_{\mathcal{L}}=0,
$$

for all open subset $U^{\prime} \subset U$ with piecewise smooth boundary, where we recall that $\varphi=\phi \circ \phi_{X}^{-1}$.

Formula (2) is also used to prove the Noether theorem. Let a Lie group $G$ act on $Y$ by $\pi_{X Y}$-bundle automorphisms and assume that the Lagrangian density $\mathcal{L}: J^{1} Y \rightarrow \Lambda^{n+1} X$ is $G$-equivariant. Then, considering only variations along the Lie group action, and restricting the action functional to an arbitrary open subset $U^{\prime} \subset U$ with piecewise smooth boundary, formula (2) shows that a solution $\varphi$ of the covariant Euler-Lagrange equation (1) verifies

$$
\int_{\partial \phi_{X}\left(U^{\prime}\right)}\left(j^{1} \varphi\right)^{*} J^{\mathcal{L}}(\xi)=0 \quad \text { for all } \xi \in \mathfrak{g},
$$

and all $U^{\prime} \subset U$, where $J^{\mathcal{L}}(\xi):=\mathbf{i}_{j^{1} \xi_{Y}} \Theta_{\mathcal{L}}: J^{1} Y \rightarrow \mathfrak{g}^{*} \otimes \Lambda^{n} J^{1} Y$ is the Lagrangian covariant momentum map associated to $\mathcal{L}$ and $\xi_{Y}$ is the infinitesimal generator of the Lie group action.

2.3. Nonsmooth variational multisymplectic geometry. The extension of the previous variational formulation to the case of fields with singularities has been carried out in Fetecau et al. [41]. In this context, one needs to introduce a codimension 1 submanifold $D \subset U$, called the singularity submanifold, across which the field $\phi$ may have singularities. We assume that $D \cap \partial U$ is a set of measure zero in $\partial U$. The extension of the variational formulation to this case is possible by introducing appropriate configuration spaces that replace $\mathcal{C}(Y)$ in the nonsmooth case. The choice of the configuration space depends on the problem treated. We recall below, from Fetecau et al. [41], three appropriate configuration spaces (the third one being a slight generalization of the one considered in Fetecau et al. [41]).

(a) Continuous and nonsmooth. Assume that the singularity submanifold $D$ separates the interior of $U$ in two disjoint open subsets $U^{+}$and $U^{-}$and that the 
fields are continuous on $U$, but smooth only on $U \backslash D$. Such a situation arises, for example, in rigid body dynamics with impact or in the propagation of singular surfaces within a continuum medium. The configuration space is the set

$$
\begin{gathered}
\mathcal{C}^{a}=\left\{\phi: U \rightarrow Y \mid \pi_{X Y} \circ \phi=\phi_{X}: U \rightarrow X\right. \text { is a smooth embedding, } \\
\left.\phi \text { is } C^{0} \text { in } U \text { and of class } C^{2} \text { in } U \backslash D\right\} .
\end{gathered}
$$

(b) Discontinuous without separation. The fields are not continuous at the singularity subset $D$, but they are required to map $D$ into a single spatial surface at each time (no separation). Such a situation arises in the case of propagation of free surfaces in fluids or interaction of an elastic body and a fluid. The configuration space is

$$
\begin{gathered}
\mathcal{C}^{b}=\left\{\phi: U \rightarrow Y \mid \pi_{X Y} \circ \phi=\phi_{X}: U \rightarrow X\right. \text { is a smooth embedding, } \\
\left.\phi \text { is } C^{2} \text { in } U^{+} \cup U^{-} \text {and } \overline{\phi^{+}}(D)=\overline{\phi^{-}}(D)=\phi(D)\right\},
\end{gathered}
$$

where $\phi^{ \pm}:=\left.\phi\right|_{U^{ \pm}}$and $\overline{\phi^{ \pm}}$denotes its continuous extension to the closure of its domain.

(c) Discontinuous with separation. This situation arises in the case of collisions of two elastic bodies. The interior of $U$ consists of two disjoint subsets $U_{1}$ and $U_{2}$, corresponding to the two bodies. The singularity submanifold $D$ is interpreted here as the spacetime contact set and therefore corresponds to two distinct hypersurfaces $D_{1} \subset \partial U_{1}$ and $D_{2} \subset \partial U_{2}$ in the reference configuration. The configuration space is

$$
\begin{gathered}
\mathcal{C}^{c}=\left\{\phi: U \rightarrow Y \mid \pi_{X Y} \circ \phi=\phi_{X}: U \rightarrow X\right. \text { is a smooth embedding, } \\
\left.\phi \text { is } C^{0} \text { in } U_{1} \cup U_{2} \text { and } \overline{\phi_{1}}\left(D_{1}\right)=\overline{\phi_{2}}\left(D_{2}\right)\right\} .
\end{gathered}
$$

Variational principle in the nonsmooth setting. Denote by $\mathcal{C}^{\text {ns }}$ (ns for nonsmooth) any of the configuration spaces $\mathcal{C}^{a}, \mathcal{C}^{b}, \mathcal{C}^{c}$. As explained in Fetecau et al. [41], $\mathcal{C}^{a}, \mathcal{C}^{b}, \mathcal{C}^{c}$ are infinite-dimensional smooth manifolds and this enables the use of differential calculus on manifolds of mappings, as required by variational principles. Given a Lagrangian density $\mathcal{L}: J^{1} Y \rightarrow \Lambda^{n+1} X$, the action functional is defined on $\mathcal{C}^{n s}$ exactly as in the smooth case, namely, $\mathfrak{S}^{n s}(\phi)=\int_{U_{X}} \mathcal{L}\left(j^{1}(\phi \circ\right.$ $\left.\phi_{X}^{-1}\right)$ ), where $U_{X}:=\phi_{X}(U)$. The derivative of $\mathfrak{S}^{n s}$ is given by (see Fetecau et al. [41]),

$$
\begin{aligned}
\mathbf{d} \mathfrak{S}^{n s}(\phi) \cdot(V \circ \phi)= & -\int_{U_{X}^{-} \cup U_{X}^{+}}\left(j^{1} \varphi\right)^{*} \mathbf{i}_{j^{1} V} \Omega_{\mathcal{L}}+\int_{D_{X}} \llbracket\left(j^{1} \varphi\right)^{*} \mathbf{i}_{j^{1} V} \Theta_{\mathcal{L}} \rrbracket \\
& +\int_{\partial U_{X} \backslash D_{X}}\left(j^{1} \varphi\right)^{*} \mathbf{i}_{j^{1} V} \Theta_{\mathcal{L}}, \quad \forall V \in \mathfrak{X}(Y),
\end{aligned}
$$


where $U_{X}^{ \pm}:=\phi_{X}\left(U^{ \pm}\right)$and $D_{X}:=\phi_{X}\left(D^{ \pm}\right)$, which generalizes (2) to the nonsmooth setting. Formula (9) is obtained by splitting the action integral $\int_{U_{X}}$ into $\int_{U_{X}^{+}}+\int_{U_{X}^{-}}$, and observing a jump term (the second summand) arising from the different orientations of $D_{X}$ when using the Stokes theorem. Given two $n$ forms $\alpha^{ \pm} \in \Omega^{n}\left(\overline{U_{X}^{ \pm}}\right)$, the associated jump is defined by

$$
\llbracket \alpha \rrbracket(x):=\alpha^{+}(x)-\alpha^{-}(x), \quad x \in D_{X} .
$$

In (9), the submanifold $D_{X}$ has the orientation of the boundary orientation of $\partial U_{X}^{+}$.

In local coordinates, writing $V=V^{\nu}\left(\partial / \partial x^{\nu}\right)+V^{A}\left(\partial / \partial y^{A}\right)$, formula (9) reads

$$
\begin{aligned}
\mathbf{d} S^{n s}(\phi) \cdot(V \circ \varphi)= & \int_{U_{X}^{+} \cup U_{X}^{-}}\left(\frac{\partial L}{\partial \varphi^{A}}-\partial_{\mu} \frac{\partial L}{\partial \varphi^{A}, \mu}\right) V^{A} d^{n+1} x \\
& +\int_{U_{X}^{+} \cup U_{X}^{-}}\left(\frac{\partial L}{\partial x^{\gamma}}+\frac{d}{d x^{\mu}}\left(\frac{\partial L}{\partial \varphi^{A}, \mu} \varphi^{A}{ }_{, \gamma}\right)-\frac{d}{d x^{\gamma}} L\right) V^{\gamma} d^{n+1} x \\
& +\int_{\partial U_{X} \backslash D_{X}}\left(\frac{\partial L}{\partial \varphi^{A}{ }_{, \mu}} V^{A} d^{n} x_{\mu}\right) \\
& +\int_{\partial U_{X} \backslash D_{X}}\left(L \delta_{v}^{\mu}-\frac{\partial L}{\partial \varphi^{A}, \mu} \varphi^{A}{ }_{, \nu}\right) V^{v} d^{n} x_{\mu} \\
& +\int_{D_{X}} \llbracket \frac{\partial L}{\partial \varphi^{A}, \mu} V^{A} d^{n} x_{\mu} \rrbracket \\
& +\int_{D_{X}} \llbracket\left(L \delta_{v}^{\mu}-\frac{\partial L}{\partial \varphi^{A}{ }_{, \mu}} \varphi^{A}{ }_{, \nu}\right) V^{v} d^{n} x_{\mu} \rrbracket
\end{aligned}
$$

From (9) it follows that $\phi$ is a critical point of $\mathfrak{S}^{n s}$ on $\mathcal{C}^{n s}$ with respect to variations with compact support in $U$ if and only if $\phi$ verifies the covariant EulerLagrange equations on $U_{X}^{-} \cup U_{X}^{+}$, the jump conditions on $D_{X}$, and the boundary conditions away from $D_{X}$, that is,

$$
\int_{D_{X}} \llbracket\left(j^{1} \varphi\right)^{*} \mathbf{i}_{j^{1} V} \Theta_{\mathcal{L}} \rrbracket=0, \quad \int_{\partial U_{X} \backslash D_{X}}\left(j^{1} \varphi\right)^{*} \mathbf{i}_{j^{1} V} \Theta_{\mathcal{L}}=0, \quad \forall V \in \mathfrak{X}(Y) .
$$

We refer to Fetecau et al. [41] for the application of this variational approach to several examples in nonsmooth continuum mechanics and for the physical interpretation of the jump conditions (17). In Section 3, we include impenetrability constraints in this geometric setting, in order to give a variational formulation of contact mechanics. This is achieved by combining 
the aforementioned variational multisymplectic treatment with the generalized Lagrange multiplier approach for constrained optimization problems, as formulated, for example, in Clarke [18], Rockafellar and Wets [120], Dontchev and Rockafellar [31].

2.4. Conservation laws in the nonsmooth setting. In this subsection we extend the Noether theorem and the multisymplectic formula to the nonsmooth multisymplectic setting recalled above.

Noether theorem in the nonsmooth setting. Let a Lie group $G$ act on $Y$ by $\pi_{X Y^{-}}$ bundle automorphisms and assume that the Lagrangian density $\mathcal{L}: J^{1} Y \rightarrow$ $\Lambda^{n+1} X$ is $G$-equivariant. This action induces a $G$-action on $\mathcal{C}^{n s}$. Then, as in the smooth case, the action functional $\mathfrak{S}^{n s}$, restricted to any $U^{\prime} \subset U$, is $G$-invariant. As a consequence of (9) we get

$$
\begin{aligned}
& \int_{\phi_{X}\left(U^{\prime}\right) \cup \phi_{X}\left(U^{\prime+}\right)}\left(j^{1} \varphi\right)^{*} \mathbf{i}_{j^{1} \xi_{Y}} \Omega_{\mathcal{L}}+\int_{\phi_{X}\left(D^{\prime}\right)} \llbracket\left(j^{1} \varphi\right)^{*} \mathbf{i}_{j^{1} \xi_{Y}} \Theta_{\mathcal{L}} \rrbracket \\
& \quad+\int_{\partial \phi_{X}\left(U^{\prime}\right) \backslash \phi_{X}\left(D^{\prime}\right)}\left(j^{1} \varphi\right)^{*} \mathbf{i}_{j^{1} \xi_{Y}} \Theta_{\mathcal{L}}=0,
\end{aligned}
$$

for all $\xi \in \mathfrak{g}$ and for all open subsets $U^{\prime} \subset U$ with piecewise smooth boundary, where $D^{\prime}:=D \cap U^{\prime}$. Therefore, if $\phi$ is a critical point of the action functional $\mathfrak{S}^{n s}$ on $\mathcal{C}^{n s}$, then the first term vanishes and $\phi$ verifies

$$
\int_{\phi_{X}\left(D^{\prime}\right)} \llbracket\left(j^{1} \varphi\right)^{*} J^{\mathcal{L}}(\xi) \rrbracket+\int_{\partial \phi_{X}\left(U^{\prime}\right) \backslash \phi_{X}\left(D^{\prime}\right)}\left(j^{1} \varphi\right)^{*} J^{\mathcal{L}}(\xi)=0,
$$

for all $\xi \in \mathfrak{g}$ and for all open subsets $U^{\prime} \subset U$ with piecewise smooth boundary. This is a generalization of the Noether theorem to the nonsmooth context. Evidently, in the particular case when $U^{\prime} \cap D=\varnothing$, it recovers the Noether theorem (5).

Note that the jump condition (17), which is part of the criticality condition on $\phi$, does not necessarily imply vanishing of the first term in (19) in general. However, this is possible in some particular situations; for example, in the case of the configuration space $\mathcal{C}^{a}$, or if $U^{\prime}=U$, in which case $D^{\prime}=D$.

Multisymplectic form formula in the nonsmooth setting. Using the arguments in Marsden et al. [82, Theorem 4.1], we extend the multisymplectic form formula (4) to the nonsmooth setting by making use of the formula (9). We get that if $\phi$ is a critical point of the action functional $\mathfrak{S}^{n s}$ on the manifold $\mathcal{C}^{n s}$ and if $V, W$, are solutions of the first variation equations at $\phi$, then they verify the formula

$$
\int_{\phi_{X}\left(D^{\prime}\right)} \llbracket\left(j^{1} \varphi\right)^{*} \mathbf{i}_{j^{1} V} \mathbf{i}_{j^{1} W} \Omega_{\mathcal{L}} \rrbracket+\int_{\partial \phi_{X}\left(U^{\prime}\right) \backslash \phi_{X}\left(D^{\prime}\right)}\left(j^{1} \varphi\right)^{*} \mathbf{i}_{j^{1} V} \mathbf{i}_{j^{1} W} \Omega_{\mathcal{L}}=0,
$$


for all open subsets $U^{\prime} \subset U$ with piecewise smooth boundary. More details are given below in the nonsmooth case with constraints.

\section{Variational multisymplectic formulation of nonsmooth mechanics with constraints}

We develop a variational approach to treat nonsmooth problems in which the singularities in the fields are due to constraints, such as impacts with a rigid plate or collision between two bodies. If the constraints on the fields define a submanifold of the configuration space $\mathcal{C}^{n s}$, the critical condition can be obtained either by computing directly the critical fields of the action $\mathfrak{S}^{n s}$ restricted to this submanifold, or by using the standard Lagrange multiplier approach. In view of the discretization developed in the next section, it turns out that it is preferable to treat these problems directly with the tools developed for more general constraints via the concept of normal cone, which yields a generalization of the Lagrange multiplier theorem (see for example, Clarke [18], Rockafellar and Wets [120], Dontchev and Rockafellar [31]).

3.1. Generalized Lagrange multiplier approach. We review the generalized Lagrange multiplier theorem for optimization problems over a constraint subset $C$. We recall the relevant definitions (see, for example, Rockafellar and Wets [120, Definitions 6.1 and 6.3]).

Let $A \subset \mathbb{R}^{p}$. A vector $v \in \mathbb{R}^{p}$ is tangent to $A \subset \mathbb{R}^{p}$ at $a \in A$, written $v \in T_{A}(a)$, if there exists a sequence $a_{k} \rightarrow a$ with $a_{k} \in A$ and a sequence $\tau_{k} \rightarrow 0, \tau_{k}>0$, such that $\lim _{k \rightarrow \infty}\left(a_{k}-a\right) / \tau_{k} \rightarrow v$. A vector $v \in \mathbb{R}^{p}$ is regular normal to $A \subset \mathbb{R}^{p}$ at $a$, written $v \in \widehat{N}_{A}(a)$, if $\left\langle v, a^{\prime}-a\right\rangle \leqslant o\left(\left|a^{\prime}-a\right|\right)$ for $a^{\prime} \in A$. A vector $v \in \mathbb{R}^{p}$ is normal to $A$ at $a$, written $v \in N_{A}(a)$, if there are sequences $a_{k} \longrightarrow a, a_{k} \in A$, and $v_{k} \rightarrow v$ with $v_{k} \in \widehat{N}_{A}\left(a_{k}\right)$. Note that $0 \in T_{A}(a), N_{A}(a)$ and that the defining inequality above means that

$$
\limsup _{a^{\prime} \rightarrow a, a^{\prime} \in A, a^{\prime} \neq a} \frac{\left\langle v, a^{\prime}-a\right\rangle}{\left|a^{\prime}-a\right|} \leqslant 0 .
$$

By definition, if $a \notin A$, then $N_{A}(a)=\varnothing$. If $a \in \operatorname{int}(A)$, then $N_{A}(a)=\{0\}$. It is known that $T_{A}(a), N_{A}(a), \widehat{N}_{A}(a)$ are closed cones (see Rockafellar and Wets [120, Propositions 6.2 and 6.5]). Moreover, $\widehat{N}_{A}(a)=\left\{v \in \mathbb{R}^{p} \mid\langle v, w\rangle \leqslant 0, \forall w \in\right.$ $\left.T_{A}(a)\right\}$ is convex and $N_{A}(a)=\lim \sup _{a^{\prime} \rightarrow a, a^{\prime} \in A} \widehat{N}_{A}\left(a^{\prime}\right) \supset \widehat{N}_{A}(a)$.

The cones $T_{A}(a), N_{A}(a), \widehat{N}_{A}(a)$ are, respectively, called the tangent, normal, and regular normal cone to $A$ at $a$. If $A \subset \mathbb{R}^{p}$ is convex we have (Rockafellar and 
Wets [120, Theorem 6.9]):

$$
\begin{gathered}
T_{A}(a)=\overline{\left\{v \in \mathbb{R}^{p} \mid \exists \epsilon>0, a+\epsilon v \in A\right\}}, \\
N_{A}(a)=\left\{v \in \mathbb{R}^{p} \mid\left\langle v, a^{\prime}-a\right\rangle \leqslant 0, \forall a^{\prime} \in A\right\} .
\end{gathered}
$$

So, every normal to a convex set is regular normal.

We now recall the fundamental connection between variational geometry and optimality conditions (see, for example, Rockafellar and Wets [120, Theorem 6.12] and Dontchev and Rockafellar [31, Theorem 2A.6]).

THEOREM 1. Consider the problem of minimizing a differentiable function $f$ : $\mathbb{R}^{p} \rightarrow \mathbb{R}$ over a subset $C \subset \mathbb{R}^{p}$. A necessary condition for $m \in C$ to be locally optimal is $-\nabla f(m) \in \widehat{N}_{C}(m)$, which implies $-\nabla f(m) \in N_{C}(m)$. When $C$ and $f$ are convex, this condition is sufficient for $m$ to be globally optimal.

If $C$ is the preimage of a convex set by a continuously differentiable function, we have the following theorem which generalizes the standard Lagrange multiplier approach (see, for example, Rockafellar and Wets [120, Theorem 6.12] and Dontchev and Rockafellar [31, Theorem 2A.8]).

THEOREM 2 (Lagrange multiplier rule). Let $Q \subset \mathbb{R}^{p}$ and $P \subset \mathbb{R}^{s}$ be nonempty, closed, convex sets, and consider $C:=g^{-1}(P)$, where $g: Q \subseteq \mathbb{R}^{p} \rightarrow \mathbb{R}^{s}$ is a continuously differentiable function. Assume that $m \in C$ satisfies the following condition:

$$
\nexists \lambda \in N_{P}(g(m)) \subset \mathbb{R}^{s}, \lambda \neq 0 \text {, such that }-\lambda \cdot \nabla g(m) \in N_{Q}(m) \subset \mathbb{R}^{p} .
$$

Then $N_{C}(m)=\left\{\lambda \cdot \nabla g(m)+N_{Q}(m) \mid \lambda \in N_{P}(g(m))\right\}$. Therefore, if $f: Q \subseteq$ $\mathbb{R}^{p} \rightarrow \mathbb{R}$ is a differentiable function and $m$ is a local minimum of $f$ relative to $C$, then there exists $\lambda \in N_{P}(g(m)) \subset \mathbb{R}^{p}$ such that

$$
-[\nabla f(m)+\lambda \cdot \nabla g(m)] \in N_{Q}(m) .
$$

REMARK 1. Recall that when $P:=\left\{\left(d^{1}, \ldots, d^{s}\right) \in \mathbb{R}^{s} \mid d^{1} \leqslant 0, \ldots, d^{s} \leqslant 0\right\}$, then $\lambda=\left(\lambda_{1}, \ldots, \lambda_{s}\right) \in N_{P}(x)$ means that $\lambda_{i}=0$ when $x^{i}<0$ and $\lambda_{i} \geqslant 0$ when $x^{i}=0$. Thus, if $Q=\mathbb{R}^{p}$, then $N_{Q}(m)=\{0\}$, and condition (22) reads explicitly $\nabla f(m)=-\sum_{i=1}^{s} \lambda_{i} \nabla g^{i}(m)$, where $\lambda_{i}=0$ if $g^{i}(m)<0$ and $\lambda_{i} \geqslant 0$ if $g^{i}(m)=0$.

3.2. Nonsmooth variational mechanics with constraints. Assume that the fiber bundle $\pi_{X Y}: Y \rightarrow X$ is trivial, that is, $Y=X \times M$ and $\pi_{X Y}$ is the projection on the first factor. Let $N:=\operatorname{dim} M$. Write $\phi=\left(\phi_{X}, \phi_{M}\right)$ and identify $\varphi=\phi \circ \phi_{X}^{-1}$ 
with $\phi_{M} \circ \phi_{X}^{-1}$. Given a singularity submanifold $D \subset U$ and a Lagrangian density $\mathcal{L}$, consider, as before, the action functional $\mathfrak{S}^{n s}: \mathcal{C}^{n s} \rightarrow \mathbb{R}$. Assume that the spatial constraint on the dynamics is given by a subset $C \subset M$, that is, we have the following constraint on the field

$$
\phi_{M}(U) \subset C .
$$

This constraint represents, for example, impenetrability in contact mechanics. In addition, singularities in the field may appear at spacetime points for which the field values belong to the boundary $\partial C$ of $C$. This is described by the constraint

$$
\phi_{M}(u) \in \partial C \Longrightarrow u \in D
$$

which does not exclude the possible presence of singularities when $\phi(u) \notin \partial C$. For example, this occurs if a singularity propagates in the body after impact (see Fetecau et al. [41]).

If singularities only appear at the spacetime points for which the field values belong to the boundary of $C$, then the constraint on the fields reads

$$
\phi_{M}(U) \subset C \text { and } \phi_{M}(u) \subset \partial C \quad \Longleftrightarrow u \in D .
$$

3.2.1. Necessary conditions for criticality under constraints. Endow $U, X$, and $M$ with Riemannian metrics, which then gives a natural duality pairing on the tangent space to $\mathcal{C}^{n s}$.

The jump of Radon-Nikodym derivatives on the singular set $D_{X}$ is defined in the following way. If $j: D_{X} \hookrightarrow U_{X}$ is the inclusion and $\alpha^{ \pm} \in \Omega^{n}\left(\overline{U_{X}^{ \pm}}\right)$(see (10) for the definition of $\llbracket \alpha \rrbracket), j^{*} \llbracket \alpha \rrbracket \in \Omega^{n}\left(D_{X}\right)$ is necessarily of the form

$$
j^{*} \llbracket \alpha \rrbracket=\llbracket f \rrbracket d v_{D_{X}} \quad \text { for some } f \in C^{\infty}\left(D_{X}\right),
$$

where $d v_{D_{X}}$ is the measure associated to the volume form on $D_{X}$ induced by the Riemannian volume form on $U_{X}$. If it is clear from the context, $j^{*}$ is omitted.

By Theorem 1, a necessary condition for a field $\phi$ to be optimal for $\mathfrak{S}^{\text {ns }}$ with respect to the constraint is formally given by

$$
-\left(\frac{\delta \mathfrak{S}^{n s}}{\delta \phi}\right)(x) \in N_{C}(\varphi(x)) \quad \forall x \in U_{X}
$$

Condition (27) is obtained by requesting the normal cone at $x$ associated to the constraint at the field $\phi$ to equal the normal cone associated to the spatial constraint $C \subset M$ at $\varphi(x)$. Note that equation (27) provides the fundamental link between field theory and optimization under inequality constraints. 
It characterizes all critical fields of the action functional even if they admit nonsmooth singularities (see Theorem 3 below).

Although a general treatment is possible, we assume, for simplicity, that $M=$ $\mathbb{R}^{p}$, endowed with the standard inner product, and that $C$ is the closure of an open subset of $M$. Let us also assume that $C=(-\psi)^{-1}(P)$, where the constraint functions

$$
\psi=\left(\psi^{1}, \ldots, \psi^{s}\right): \mathbb{R}^{p} \rightarrow \mathbb{R}^{s}
$$

are continuously differentiable and the domain $P$ is

$$
P:=\left\{\left(d^{1}, \ldots, d^{s}\right) \in \mathbb{R}^{s} \mid d^{1} \leqslant 0, \ldots, d^{s} \leqslant 0\right\} .
$$

The normal cone is $N_{C}(\varphi(x))=\left\{\lambda_{1}(x) \nabla \psi^{1}(\varphi(x))+\cdots+\lambda_{s}(x) \nabla \psi^{s}(\varphi(x)) \mid\right.$ $\lambda(x) \in N_{P}(-\psi(\varphi(x))\}$, for all $x \in U_{X}$. Suppose that (21) holds for $\lambda$ and $\psi$. Thus, we have the following cases.

(i) If $x \in U_{X}^{-} \cup U_{X}^{+}$, then from (23) and (24) we have $\varphi(x) \in \operatorname{int}(C)$. Since we assumed that $C$ is the closure of an open subset of $M$, the normal cone to $C$ at $\varphi(x)$ in $M$ reduces to zero. In this case, using (11) and (12), the vertical and horizontal components of (27) yield, respectively, the covariant Euler-Lagrange equations on $U_{X}^{-} \cup U_{X}^{+}$

$$
\frac{\partial L}{\partial \varphi^{A}}-\partial_{\mu} \frac{\partial L}{\partial \varphi^{A}, \mu}=0 \text { for all } A=1, \ldots, N,
$$

together with the balance of energy and configurational forces (3) on $U_{X}^{-} \cup U_{X}^{+}$.

(ii) If $x \in D_{X}$, then from (23) and (24) we can have $\varphi(x) \in \partial C$ or $\varphi(x) \in \operatorname{int}(C)$. Using (15), the vertical component of (27) yields the condition

$$
\int_{D_{X}} \llbracket\left(\frac{\partial L}{\partial \varphi^{A}, \mu}(x) N_{\mu}(x)-\lambda(x) \cdot \psi_{, A}(\varphi(x))\right) V^{A}(\varphi(x)) \rrbracket d v_{D_{X}}=0, \quad \forall V \in T_{\phi} \mathcal{C}^{n s},
$$

where $\lambda(x) \in N_{P}(-\psi(\varphi(x))) ; N_{\mu}$ is the normal vector field to the singularity submanifold and $d v_{D_{X}}$ is the volume form induced on $D_{X}$. If $\varphi(x) \in \operatorname{int}(C)$, then $\lambda(x)=0$. Using (16), the horizontal component of (27) yields, in this case, the condition

$$
\llbracket L \delta_{v}^{\mu}-\frac{\partial L}{\partial \varphi_{, \mu}^{A}} \varphi_{, v}^{A} \rrbracket N_{\mu}=0 \quad \text { on } D_{X} \quad \text { for all } v=0,1, \ldots, n .
$$

If $\mathcal{C}^{n s}=\mathcal{C}^{a}$, the first condition in (ii) is equivalent to

$$
\llbracket \frac{\partial L}{\partial \varphi^{A}{ }_{, \mu}}(x) \rrbracket N_{\mu}(x)=\lambda(x) \cdot \psi_{, A}(\varphi(x)) \quad \text { on } D_{X}, \quad \forall A=1, \ldots, N .
$$


THEOREM 3. Consider a Lagrangian density $\mathcal{L}: J^{1} Y \rightarrow \Lambda^{n+1} X$ and assume the same hypotheses as above on $\pi_{X Y}: Y \rightarrow X, C$, and (21). Then $\phi$ is a critical point of $\mathfrak{S}^{n s}$ relative to the constraints (23) and (24) if and only if

- Away from the singularity, the field $\phi$ satisfies the covariant Euler-Lagrange equations on $U_{X}^{-} \cup U_{X}^{+}$,

$$
\frac{\partial L}{\partial \varphi^{A}}-\partial_{\mu} \frac{\partial L}{\partial \varphi^{A}, \mu}=0, \quad \forall A=1, \ldots, N,
$$

together with the balance of energy and configurational forces (3) on $U_{X}^{-} \cup U_{X}^{+}$.

- At the singularity $D_{X}$, the field $\phi$ verifies the following conditions:

(a) the vertical jump condition: for all vector fields $V$ on $Y$, we have

$$
\int_{D_{X}} \llbracket\left(\frac{\partial L}{\partial \varphi^{A}, \mu}(x) N_{\mu}(x)-\lambda(x) \cdot \psi_{, A}(\varphi(x))\right) V^{A}(\varphi(x)) \rrbracket d v_{D_{X}}=0,
$$

where the Lagrange multiplier $\lambda(x) \in N_{P}(-\psi(\varphi(x)))$.

(b) the horizontal jump condition on $D_{X}$

$$
\llbracket L \delta_{v}^{\mu}-\frac{\partial L}{\partial \varphi^{A}, \mu} \varphi^{A}{ }_{, v} \rrbracket N_{\mu}=0, \quad \forall v=0,1, \ldots, n .
$$

- On the boundary $\partial U_{X} \backslash D_{X}$, the field $\phi$ verifies the following conditions:

(c) for all $A=1, \ldots, N$, we have

$$
\frac{\partial L}{\partial \varphi^{A}, \mu} V^{A} N_{\mu}=0
$$

(d) for all $v=0, \ldots, n$, we have

$$
\left(L \delta_{v}^{\mu}-\frac{\partial L}{\partial \varphi_{, \mu}^{A}} \varphi_{, v}^{A}\right) N_{\mu}=0 .
$$

REMARK 2. Theorem 3 can be generalized to the case when $C$ is not the closure of an open subset; for example, if $C$ is a submanifold with boundary of $M$. In this case, Lagrange multipliers also appear in the covariant Euler-Lagrange equations. 
REMARK 3. For classical nonsmooth mechanics, Theorem 3 recovers the variational setting developed in Fetecau et al. [42] by choosing $\mathcal{C}^{n s}=\mathcal{C}^{a}$, $U=[0,1], D=\left\{\tau_{i}\right\}, C$ an open subset of $M$ with smooth boundary, and the constraint (25). In this case, (25) defines a smooth manifold (see Fetecau et al. [42, Section 2.1]) and standard variational calculus can be used. In this special case, for all $m \in \partial C$, the normal cone $N_{C}(m)$ is a half space in $N_{\partial C}(m)=\left(T_{m} C\right)^{\perp}$.

More generally, in the field theoretic context, we note that if the constraint (23)-(24) or (25) define a manifold, then standard variational calculus can also be used to characterize the critical points of $\mathfrak{S}^{n s}$.

REMARK 4. In Sections 4 and 5 below, we consider in detail the discretization of two $1+1$-dimensional examples, namely, the case of the impact of a 1-dimensional elastic bar on a rigid plane and the impact of two 1-dimensional elastic bars. In the first case, the constraint has the form (24), since a singularity in the field may not only appear at the spacetime location of the impact but also as a propagating singularity through the body after impact. For the second case, the constraint is also of the form (24), but the configuration space involves a combination of both $\mathcal{C}^{a}$ and $\mathcal{C}^{c}$, because of the separation of the two bars before and after the contact.

3.2.2. Noether theorem. Let a Lie group $G$ act on $Y$ by $\pi_{X Y}$-bundle automorphisms and assume that the Lagrangian density $\mathcal{L}: J^{1} Y \rightarrow \Lambda^{n+1} X$ is $G$-equivariant. Then the Noether theorem (18) holds for the induced $G$-action on $\mathcal{C}^{n s}$ and for all $\phi \in \mathcal{C}^{n s}$, verifying the constraint or not. Therefore, if $\phi$ verifies the hypotheses of Theorem 3 and the covariant Euler-Lagrange equations, then it also verifies (19). If $\mathcal{C}^{n s}=\mathcal{C}^{a}$ or if $U^{\prime}=U$, then by using the vertical jump condition in Theorem 3 the first term in (19) can be rewritten in terms of Lagrange multipliers.

Note that if a field $\phi \in \mathcal{C}^{n s}$ verifies the constraints (23) and (24), then the new field obtained by applying the Lie group action still belongs to $\mathcal{C}^{n s}$ but it no longer verifies the constraints (23) and (24), in general.

3.2.3. Multisymplectic form formula. Given $\phi \in \mathcal{C}^{n s}$ and two vector fields $V, W$ on $Y$, we have the formula

$$
\begin{aligned}
0= & \mathbf{d}^{2} \mathfrak{S}^{n s}(\phi)(V \circ \varphi, W \circ \varphi) \\
= & -\int_{\phi_{X}\left(U^{\prime}\right) \cup \phi_{X}\left(U^{\prime+}\right)}\left(j^{1} \varphi\right)^{*}\left(\boldsymbol{\xi}_{j^{1} V} \mathbf{i}_{j^{1} W} \Omega_{\mathcal{L}}-\boldsymbol{f}_{j^{1} W} \mathbf{i}_{j^{1} V} \Omega_{\mathcal{L}}-\mathbf{i}_{\left[j^{1} V, j^{1} W\right]} \Omega_{\mathcal{L}}\right) \\
& +\int_{\partial \phi_{X}\left(U^{\prime}\right) \backslash \phi_{X}\left(D^{\prime}\right)}\left(j^{1} \varphi\right)^{*} \mathbf{i}_{j^{1} V} \mathbf{i}_{j^{1} W} \Omega_{\mathcal{L}}+\int_{\partial \phi_{X}\left(D^{\prime}\right)} \llbracket\left(j^{1} \varphi\right)^{*} \mathbf{i}_{j^{1} V} \mathbf{i}_{j^{1} W} \Omega_{\mathcal{L}} \rrbracket,
\end{aligned}
$$


which is obtained by extending to the nonsmooth case the arguments in Marsden et al. [82, Theorem 4.1].

Suppose $\phi \in \mathcal{C}^{n s}$ satisfies the constraints (23) and verifies the conditions of Theorem 3. In particular, $\phi$ verifies $\left(j^{1} \varphi\right)^{*} \mathbf{i}_{j^{1} Z} \Omega_{\mathcal{L}}=0$ on $U_{X}^{-} \cup U_{X}^{+}$(that is, away from contact) for all $Z \in \mathfrak{X}(Y)$. We say that $V \in \mathfrak{X}(Y)$, with $T \pi_{X Y} \circ V \in \mathfrak{X}(X)$, is a first variation at $\phi$ on $U_{X}^{-} \cup U_{X}^{+}$if its flow $\eta^{\varepsilon}$ is such that $\phi^{\varepsilon}:=\eta^{\varepsilon} \circ \phi$ is a solution of $\left(j^{1} \varphi^{\varepsilon}\right)^{*} \mathbf{i}_{j^{1} Z} \Omega_{\mathcal{L}}=0$ on $U_{X}^{-} \cup U_{X}^{+}$, for all $Z \in \mathfrak{X}(Y)$ and all $\varepsilon$ in an open interval containing $0 \in \mathbb{R}$, where $\phi_{X}^{\varepsilon}:=\pi_{X Y} \circ \phi^{\varepsilon}: U \rightarrow X$ is an embedding and $\varphi^{\varepsilon}:=\phi^{\varepsilon} \circ\left(\phi_{X}^{\varepsilon}\right)^{-1}: \phi_{X}^{\varepsilon}(U) \rightarrow Y$ is a section of $\pi_{X Y}: Y \rightarrow X$ restricted to $\phi_{X}^{\varepsilon}(U) \subset X$. Such a first variation thus verifies $\left(j^{1} \varphi\right)^{*} \boldsymbol{\xi}_{j^{1} V} \mathbf{i}_{j^{1} Z} \Omega_{\mathcal{L}}=0$, for all $Z \in \mathfrak{X}(Y)$. From (35), we thus obtain the following statement.

Let $\phi$ verify the conditions of Theorem 3. Let $V, W \in \mathfrak{X}(Y)$ be such that $T \pi_{X Y} \circ$ $V, T \pi_{X Y} \circ W \in \mathfrak{X}(X)$ are first variations at $\phi$ on $U_{X}^{-} \cup U_{X}^{+}$. Then $\phi, V, W$ verify (20).

\section{Multisymplectic variational integrator for nonsmooth mechanics with constraints}

In this section, we start the development of a discrete counterpart of the variational multisymplectic framework obtained for nonsmooth field theory with constraints in Section 3.2. The multisymplectic integrators for such problems are thus obtained by combining the generalized Lagrange multiplier approach for general constrained problems, presented in Section 3.1, with the theory of multisymplectic integrators. We illustrate our approach by focusing on a $1+1$ dimensional spacetime, with a discretization based on a rectangular mesh. Our setting is, however, easily formulated for higher-dimensional spacetimes and other classes of spacetime discretizations.

First, we quickly review, from Marsden et al. [82] and Lew et al. [80, 81], some facts about multisymplectic variational integrators for smooth unconstrained problems. Then, we develop our approach for two different classes of constrained problems, using the generalized Lagrange multiplier approach. These two classes correspond to the problems of the impact of a 1-dimensional elastic body with a rigid plate and of the impact of two 1-dimensional elastic bodies.

4.1. Multisymplectic variational integrators. We present separately the discrete analogues of the mathematical concepts in classical field theory.

Discrete bundles and configurations. Consider a trivial bundle $Y=X \times M \rightarrow X$, with $X=[0, T] \times[0, L]$, and the discrete parameter space given by

$$
U_{d}=\{0, \ldots, N\} \times\{0, \ldots, A\},
$$


where $N$ and $A$ are the number of temporal and spatial grid points, respectively. We denote by $\square_{a}^{j}$ the rectangle given by the four pairs of indices

$$
\begin{gathered}
\square_{a}^{j}=((j, a),(j+1, a),(j, a+1),(j+1, a+1)), \\
j=0, \ldots, N-1, a=0, \ldots, A-1,
\end{gathered}
$$

and by $U_{d}^{\square}$ the set of all such rectangles.

A discrete configuration is a map $\phi_{d}: U_{d} \ni(j, a) \mapsto \phi_{d}(j, a)=: \phi_{a}^{j} \in X \times M$ such that the induced map $\phi_{X_{d}}:=\pi_{X Y} \circ \phi_{d}: U_{d} \rightarrow X$, called the discrete basespace configuration, encodes the current grid configuration. The discrete section associated with $\phi_{d}$ is defined exactly as in the continuous case by $\varphi_{d}:=\phi_{d} \circ \phi_{X_{d}}^{-1}$ : $\phi_{X_{d}}\left(U_{d}\right) \rightarrow \phi_{X_{d}}\left(U_{d}\right) \times M$, which we identify with its second component, also denoted by $\varphi_{d}: \phi_{X_{d}}\left(U_{d}\right) \rightarrow M$. Thus, the knowledge of the map $\phi_{d}$ is equivalent to the knowledge of the couple of maps $\left(\phi_{X_{d}}, \varphi_{d}\right)$.

Unlike the continuous case, in the discrete setting we need to restrict the choice of the allowed discrete base-space configurations $\phi_{X_{d}}$. This class encodes the freedom we allow on the spacetime mesh and determines the discrete horizontal variations.

For example, we can restrict to maps $\phi_{X_{d}}$ of the special form

$$
\phi_{X_{d}}(j, a)=\left(\phi_{X_{d}}^{0}(j), \phi_{X_{d}}^{1}(a)\right)=:\left(t^{j}, s_{a}\right),
$$

where $\phi_{X_{d}}^{0}(0)=0, \phi_{X_{d}}^{0}(N)=T, \phi_{X_{d}}^{1}(0)=0, \phi_{X_{d}}^{1}(A)=L$. Such a discrete basespace discretization defines $N A$ rectangles in $X$. For simplicity, we also denote by $\square_{a}^{j}$ the rectangle in $X$ with lower left corner given by $\phi_{X_{d}}(j, a)$. The choice (36) allows to vary the discrete time step (uniformly in space) and to vary the discrete space step (uniformly in time). Such a choice is not necessarily interesting in applications; it serves, however, as an illustration for this subsection and as a preparation for the next developments in Sections 4.2 and 4.3.

The first jet extension of a discrete section $\varphi_{d}$ is the map $j^{1} \varphi_{d}: \phi_{X_{d}}\left(U_{d}^{\square}\right) \rightarrow$ $\phi_{X_{d}}\left(U_{d}^{\square}\right) \times M \times M \times M \times M$ defined by

$$
j^{1} \varphi_{d}(\square):=\left(\square, \varphi_{d}\left(\square^{(1)}\right), \varphi_{d}\left(\square^{(2)}\right), \varphi_{d}\left(\square^{(3)}\right), \varphi_{d}\left(\square^{(4)}\right)\right),
$$

$\square^{(1)}=(j, a), \square^{(2)}=(j+1, a), \square^{(3)}=(j, a+1), \square^{(4)}=(j+1, a+1)$, for $\square=\square_{a}^{j}$. The discrete version of the first jet bundle is given by

$$
J^{1} Y_{d}:=\phi_{X_{d}}\left(U_{d}^{\square}\right) \times(M \times M \times M \times M) \rightarrow \phi_{X_{d}}\left(U_{d}^{\square}\right) .
$$

Discrete action functional. A discrete Lagrangian is a map $\mathcal{L}_{d}: J^{1} Y_{d} \rightarrow \mathbb{R}$, constructed such that the expression $\mathcal{L}_{a}^{j}:=\mathcal{L}_{d}\left(\square_{a}^{j}, \varphi_{a}^{j}, \varphi_{a}^{j+1}, \varphi_{a+1}^{j}, \varphi_{a+1}^{j+1}\right)$ is an approximation of the action functional restricted to $\square_{a}^{j}$, namely,

$$
\mathcal{L}_{a}^{j} \approx \int_{\phi_{X_{d}}\left(\square_{a}^{j}\right)} \mathcal{L}\left(t, s, \varphi(t, s), \partial_{t} \varphi(t, s), \partial_{s} \varphi(t, s)\right) d t \wedge d s,
$$


where $\varphi: X \rightarrow M$ is a smooth field interpolating the discrete field values $\left(\varphi_{a}^{j}, \varphi_{a}^{j+1}, \varphi_{a+1}^{j}, \varphi_{a+1}^{j+1}\right)$. The discrete action functional associated to $\mathcal{L}_{d}$ and to a discrete configuration $\phi_{d}$ is

$$
\mathfrak{S}_{d}\left(\phi_{d}\right)=\sum_{j=0}^{N-1} \sum_{a=0}^{A-1} \mathcal{L}_{d}\left(\square_{a}^{j}, \varphi_{a}^{i}, \varphi_{a}^{j+1}, \varphi_{a+1}^{j}, \varphi_{a+1}^{j+1}\right),
$$

where we note that the dependence of $\mathfrak{S}_{d}$ on $\phi_{X_{d}}$ arises through the explicit dependence of $\mathcal{L}_{d}$ on $\square_{a}^{j}$.

Discrete vertical and horizontal variations. In the discrete setting, the horizontal and vertical variations of the discrete configuration $\phi_{d}$ are $\delta \phi_{X_{d}}$ and $\delta \varphi_{d}$, respectively. In order to make explicit the dependence of the discrete action functional on both the discrete base-space configuration and the discrete section, we often write $\mathfrak{S}_{d}\left(\phi_{X_{d}}, \varphi_{d}\right)=\sum_{j=0}^{N-1} \sum_{a=0}^{A-1} \mathcal{L}_{a}^{j}$. The total variation is

$$
\mathbf{d} \mathfrak{S}_{d}\left(\phi_{X_{d}}, \varphi_{d}\right) \cdot\left(\delta \phi_{X_{d}}, \delta \varphi_{d}\right)=D_{H} \mathfrak{S}_{d}\left(\phi_{X_{d}}, \varphi_{d}\right) \cdot \delta \phi_{X_{d}}+D_{V} \mathfrak{S}_{d}\left(\phi_{X_{d}}, \varphi_{d}\right) \cdot \delta \varphi_{d}
$$

where the two terms are the horizontal and vertical variations, respectively. To apply the variational principle and discuss boundary conditions, it is necessary to specify the boundary and interior of the nodal base space $\phi_{X_{d}}\left(U_{d}\right)$. In our case, the boundary is given by

$$
\begin{aligned}
\partial \phi_{X_{d}}\left(U_{d}\right)=\{ & \phi_{X_{d}}(0, a), \phi_{X_{d}}(N, a), \phi_{X_{d}}(j, 0), \\
& \left.\phi_{X_{d}}(j, A) \mid a=0, \ldots, A, j=0, \ldots, N\right\} .
\end{aligned}
$$

The discrete covariant Euler-Lagrange equations are obtained by requiring that the discrete action be stationary under arbitrary vertical variations vanishing at the boundary.

As already mentioned, the discrete local balance of configurational forces is obtained by requiring that the discrete action is stationary under horizontal variations vanishing at the boundary. The allowable horizontal variations depend on the chosen class of discrete base-space configurations. This choice depends on the properties one wants to preserve from the local balance of configurational forces at the discrete level.

As an example, for the class of discrete base-space configurations (36), the variation (39) of the discrete action functional relative to $\delta \phi_{X_{d}}$ and $\delta \varphi_{d}$ reads

$$
\begin{aligned}
\mathbf{d} \mathfrak{S}_{d}\left(\phi_{X_{d}}, \varphi_{d}\right) \cdot\left(\delta \phi_{X_{d}}, \delta \varphi_{d}\right) \\
\quad=\sum_{j=1}^{N-1} \sum_{a=1}^{A-1}\left(D_{1} \mathcal{L}_{a}^{j}+D_{2} \mathcal{L}_{a}^{j-1}+D_{3} \mathcal{L}_{a-1}^{j}+D_{4} \mathcal{L}_{a-1}^{j-1}\right) \cdot \delta \varphi_{a}^{j}
\end{aligned}
$$




$$
\begin{aligned}
& +\sum_{a=1}^{A-1}\left\{\left(D_{1} \mathcal{L}_{a}^{0}+D_{3} \mathcal{L}_{a-1}^{0}\right) \cdot \delta \varphi_{a}^{0}+\left(D_{2} \mathcal{L}_{a}^{N-1}+D_{4} \mathcal{L}_{a-1}^{N-1}\right) \cdot \delta \varphi_{a}^{N}\right\} \\
& +\sum_{j=1}^{N-1}\left\{\left(D_{1} \mathcal{L}_{0}^{j}+D_{2} \mathcal{L}_{0}^{j-1}\right) \cdot \delta \varphi_{0}^{j}+\left(D_{3} \mathcal{L}_{A-1}^{j}+D_{4} \mathcal{L}_{A-1}^{j-1}\right) \cdot \delta \varphi_{A}^{j}\right\} \\
& +D_{1} \mathcal{L}_{0}^{0} \cdot \delta \varphi_{0}^{0}+D_{2} \mathcal{L}_{0}^{N-1} \cdot \delta \varphi_{0}^{N}+D_{3} \mathcal{L}_{A-1}^{0} \cdot \delta \varphi_{A}^{0}+D_{4} \mathcal{L}_{A-1}^{N-1} \cdot \delta \varphi_{A}^{N} \\
& +\sum_{a=0}^{A-1} D_{t^{0}} \mathcal{L}_{a}^{0} \cdot \delta t^{0}+\sum_{j=1}^{N-1} \sum_{a=0}^{A-1}\left(D_{t^{j}} \mathcal{L}_{a}^{j}+D_{t^{j}} \mathcal{L}_{a}^{j-1}\right) \cdot \delta t^{j}+\sum_{a=0}^{A-1} D_{t^{N}} \mathcal{L}_{a}^{N-1} \cdot \delta t^{N} \\
& +\sum_{j=0}^{N-1} D_{s_{0}} \mathcal{L}_{0}^{j} \cdot \delta s_{0}+\sum_{a=1}^{N-1} \sum_{j=0}^{N-1}\left(D_{s_{a}} \mathcal{L}_{a}^{j}+D_{s_{a}} \mathcal{L}_{a-1}^{j}\right) \cdot \delta s_{a}+\sum_{j=0} D_{s_{A}} \mathcal{L}_{A-1}^{j} \cdot \delta s_{A} .
\end{aligned}
$$

According to our choice of discrete base-space configuration, we have $\phi_{X_{d}}^{0}(0)=0$, $\phi_{X_{d}}^{0}(N)=T, \phi_{X_{d}}^{1}(0)=0, \phi_{X_{d}}^{1}(A)=L$, so the terms in $\delta t^{0}, \delta t^{N}, \delta s_{0}, \delta s_{A}$ vanish.

Discrete covariant Euler-Lagrange equations and discrete balance of configurational forces. The discrete covariant Euler-Lagrange equations are obtained by requiring stationarity of the action $\mathfrak{S}_{d}$ under vertical variations vanishing at the boundary, that is,

$$
D_{V} \mathfrak{S}_{d}\left(\phi_{X_{d}}, \varphi_{d}\right) \cdot \delta \varphi_{d}=0 \text { for all } \delta \varphi_{d} \text { vanishing at the boundary. }
$$

This reads explicitly

$$
D_{1} \mathcal{L}_{a}^{j}+D_{2} \mathcal{L}_{a}^{j-1}+D_{3} \mathcal{L}_{a-1}^{j}+D_{4} \mathcal{L}_{a-1}^{j-1}=0, \quad j=1, \ldots, N-1, a=1, \ldots, A-1
$$

The discrete local balance of energy and of configurational forces are obtained by requiring stationarity of the action $\mathfrak{S}_{d}$ under horizontal variations vanishing at the boundary, that is,

$$
D_{H} \mathfrak{S}_{d}\left(\phi_{X_{d}}, \varphi_{d}\right) \cdot \delta \phi_{X_{d}}=0 \text { for all } \delta \phi_{X_{d}} \text { vanishing at the boundary. }
$$

For the choice (36), these equations are

$$
\begin{aligned}
& \sum_{a=0}^{A-1} D_{t^{j}} \mathcal{L}_{a}^{j}+D_{t^{j}} \mathcal{L}_{a}^{j-1}=0, \quad j=1, \ldots, N-1, \\
& \quad \sum_{j=0}^{N-1} D_{s_{a}} \mathcal{L}_{a}^{j}+D_{s_{a}} \mathcal{L}_{a-1}^{j}=0, \quad a=1, \ldots, A-1 .
\end{aligned}
$$


The treatment of boundary terms depends on the boundary conditions imposed on the discrete fields. If the values of the discrete fields are given on a part of the boundary, then the vertical variations vanish on this part of the boundary and the variational principle does not yield additional boundary conditions. On the part of the boundary where the discrete field is not prescribed, the variational principle yields zero-traction type boundary conditions. They are obtained by collecting all the terms proportional to $\delta \varphi_{a}^{j}$ for $(a, j)$ on that part of boundary. For such $(a, j)$, these boundary condition are written using (42), with the convention that $D_{k} \mathcal{L}_{a}^{j}=0$, whenever $(j, a) \notin U_{d}=\{0, \ldots, N\} \times\{0, \ldots, A\}$. Discrete (nonzero) traction boundary conditions can be easily handled by considering a slight modification of the discrete variational principle, as explained in Lew et al. [80].

Discrete multisymplectic form formula. To formulate the multisymplectic character of the integrator when horizontal variations are allowed, we introduce the following discrete Cartan forms on $J^{1} Y_{d}$ :

$$
\begin{aligned}
& \Theta_{\mathcal{L}_{d}}^{1}\left(\square_{a}^{j}, \varphi_{a}^{j}, \varphi_{a}^{j+1}, \varphi_{a+1}^{j}, \varphi_{a+1}^{j+1}\right):=D_{X_{a}^{j}} \mathcal{L}_{a}^{j} d X_{a}^{j}+D_{1} \mathcal{L}_{a}^{j} d \varphi_{a}^{j}, \\
& \Theta_{\mathcal{L}_{d}}^{2}\left(\square_{a}^{j}, \varphi_{a}^{j}, \varphi_{a}^{j+1}, \varphi_{a+1}^{j}, \varphi_{a+1}^{j+1}\right):=D_{X_{a}^{j+1}} \mathcal{L}_{a}^{j} d X_{a}^{j+1}+D_{2} \mathcal{L}_{a}^{j} d \varphi_{a}^{j+1}, \\
& \Theta_{\mathcal{L}_{d}}^{3}\left(\square_{a}^{j}, \varphi_{a}^{j}, \varphi_{a}^{j+1}, \varphi_{a+1}^{j}, \varphi_{a+1}^{j+1}\right):=D_{X_{a+1}^{j}} \mathcal{L}_{a}^{j} d X_{a+1}^{j}+D_{3} \mathcal{L}_{a}^{j} d \varphi_{a+1}^{j}, \\
& \Theta_{\mathcal{L}_{d}}^{4}\left(\square_{a}^{j}, \varphi_{a}^{j}, \varphi_{a}^{j+1}, \varphi_{a+1}^{j}, \varphi_{a+1}^{j+1}\right):=D_{X_{a+1}^{j+1}} \mathcal{L}_{a}^{j} d X_{a+1}^{j+1}+D_{4} \mathcal{L}_{a}^{j} d \varphi_{a+1}^{j+1},
\end{aligned}
$$

where we denoted by $X_{a}^{j}, X_{a}^{j+1}, X_{a+1}^{j}, X_{a+1}^{j+1}$ the nodes associated to the square $\square_{a}^{j}$. These discrete forms generalize the forms introduced in Marsden et al. [82]. If the base space is 1-dimensional, they recover the discrete forms defined in Marsden and West [83] for time-dependent mechanics.

Given a vector field $V_{d}$ tangent to the discrete configuration $\phi_{d}=\left(\phi_{X_{d}}, \varphi_{d}\right)$, we can define its first jet extension $j^{1} V_{d}$ which takes the set of nodes in $\square_{a}^{j}$ to the set of values of $V_{d}$ on these nodes. It is important to recall that $V_{d}$ encodes both horizontal and vertical variations, denoted by $V_{h}$ and $V_{v}$, respectively. Using these notations, we can write formulas of the type

$$
\begin{aligned}
& D_{X_{a}^{j}} \mathcal{L}_{d}\left(\square_{a}^{j}, \varphi_{a}^{j}, \varphi_{a}^{j+1}, \varphi_{a+1}^{j}, \varphi_{a+1}^{j+1}\right) \cdot\left(V_{h}\right)_{a}^{j} \\
& \quad+D_{1} \mathcal{L}_{d}\left(\square_{a}^{j}, \varphi_{a}^{j}, \varphi_{a}^{j+1}, \varphi_{a+1}^{j}, \varphi_{a+1}^{j+1}\right) \cdot\left(V_{v}\right)_{a}^{j}=\left[\left(j^{1} \phi_{d}\right)^{*}\left(\mathbf{i}_{j^{1} V_{d}} \Theta_{\mathcal{L}_{d}}^{1}\right)\right]\left(\square_{a}^{j}\right),
\end{aligned}
$$

that are useful to derive the discrete multisymplectic form formula. In the case where base-space configurations are not allowed to vary, the first term in (45) is not present and the first terms in all the discrete Cartan forms (44) are not needed. 
With these notations, the total derivative of the discrete action functional is

$$
\mathbf{d} \mathfrak{S}_{d}\left(\phi_{d}\right) \cdot V_{d}=\sum_{\square \in U_{d}^{\square}} \sum_{k \in \square}\left[\left(j^{1} \phi_{d}\right)^{*}\left(\mathbf{i}_{j^{1} V_{d}} \Theta_{\mathcal{L}_{d}}^{k}\right)\right](\square),
$$

using all allowed discrete configurations $\left(\phi_{X_{d}}, \varphi_{d}\right)$. If $\phi_{X_{d}}$ is given by (36), this formula recovers (40). When restricted to solutions of (41) and (43), the derivative of $\mathfrak{S}_{d}$ reads

$$
\mathbf{d} \mathfrak{S}_{d}\left(\phi_{d}\right) \cdot V_{d}=\sum_{\square \in U_{d}^{\square}} \sum_{k ; \square^{(k)} \in \partial U_{d}}\left[\left(j^{1} \phi_{d}\right)^{*}\left(\mathbf{i}_{j^{1} V_{d}} \Theta_{\mathcal{L}_{d}}^{k}\right)\right](\square) .
$$

Taking one exterior derivative and evaluating it on first variations $V_{d}, W_{d}$ of a solution $\phi_{d}=\left(\phi_{X_{d}}, \varphi_{d}\right)$ of (41) and (43), we get the discrete multisymplectic form formula

$$
\sum_{\square \in U_{d}^{\square}} \sum_{k ; \square(k) \in \partial U_{d}}\left[\left(j^{1} \phi_{d}\right)^{*}\left(\mathbf{i}_{j^{1} V_{d}} \mathbf{i}_{j^{1} W_{d}} \Omega_{\mathcal{L}_{d}}^{k}\right)\right](\square)=0,
$$

where $\Omega_{\mathcal{L}_{d}}^{k}, k=1, \ldots, 4$, are the Cartan 2-forms on $J^{1} Y_{d}$ defined by $\Omega_{\mathcal{L}_{d}}^{k}:=$ $-\mathbf{d} \Theta_{\mathcal{L}_{d}}^{k}$. Note that a first variation $V_{d}$ of a solution $\phi_{d}$ verifies the condition $D^{2} \mathfrak{S}_{d}\left(\phi_{d}\right)\left(V_{d}, W_{d}^{i n t}\right)=0$, for all vector fields $W_{d}^{\text {int }}$ vanishing at the boundary. In all these formulas, it is important to recall that the derivative of $\mathfrak{S}_{d}$ is taken on the space of allowed discrete configurations $\phi_{d}=\left(\phi_{X_{d}}, \varphi_{d}\right)$. In absence of horizontal variations, (47) recovers formula (5.6) in Marsden et al. [82].

Depending on the choice of the base-space configuration, it is possible to formulate the discrete multisymplectic form formula (47) on subdomains $U_{d}^{\prime}$ of $U_{d}$, the condition being that the discrete equations on $U_{d}$ imply the discrete equations on $U_{d}^{\prime}$. This always holds for the vertical equations but may be false for the horizontal equations, depending on the base-space configuration used. For this to be the case, the base-space configurations must allow independent variations of all nodes. For example, this is false for the choice (36), but it does hold in the absence of horizontal variations.

Discrete covariant momentum maps and discrete Noether theorem. We now consider only vertical symmetries, that is, group actions that act trivially on the base $X$. Let $\Phi: G \times M \rightarrow M$ be a left action of a Lie group $G$ on $M$. This action naturally induces one on the discrete jet bundle, which we choose to be trivial on $\square_{a}^{j}$ and diagonal on $\left(\varphi_{a}^{j}, \varphi_{a}^{j+1}, \varphi_{a+1}^{j}, \varphi_{a+1}^{j+1}\right)$. The associated infinitesimal generator reads

$$
\xi_{J^{1} Y_{d}}\left(\square_{a}^{j}, \varphi_{a}^{j}, \varphi_{a}^{j+1}, \varphi_{a+1}^{j}, \varphi_{a+1}^{j+1}\right):=\left(\square_{a}^{j}, \xi_{M}\left(\varphi_{a}^{j}\right), \xi_{M}\left(\varphi_{a}^{j+1}\right), \xi_{M}\left(\varphi_{a+1}^{j}\right), \xi_{M}\left(\varphi_{a+1}^{j+1}\right)\right),
$$


where $\xi_{M} \in \mathfrak{X}(M)$ is the infinitesimal generator of the action $\Phi$. We say that the discrete Lagrangian is invariant with respect to this action if $\mathcal{L}_{d} \circ \Phi_{g}^{J^{1} Y_{d}}=\mathcal{L}_{d}$, for all $g \in G$. Thus, we have the infinitesimal invariance $\mathbf{d} \mathcal{L}_{d} \cdot \xi_{J^{1} Y_{d}}=0$.

The discrete momentum maps are defined by

$$
J_{\mathcal{L}_{d}}^{k}: J^{1} Y_{d} \rightarrow \mathfrak{g}^{*}, \quad\left\langle J_{\mathcal{L}_{d}}^{k}, \xi\right\rangle:=\mathbf{i}_{\xi_{J^{1} Y_{d}}} \Theta_{\mathcal{L}}^{k}, \quad \xi \in \mathfrak{g}, k=1, \ldots, 4,
$$

so we have the formulas

$$
\left\langle J_{\mathcal{L}}^{k}\left(\square_{a}^{j}, \varphi_{a}^{j}, \varphi_{a}^{j+1}, \varphi_{a+1}^{j}, \varphi_{a+1}^{j+1}\right), \xi\right\rangle=D_{k} \mathcal{L}_{d}\left(\square_{a}^{j}, \varphi_{a}^{j}, \varphi_{a}^{j+1}, \varphi_{a+1}^{j}, \varphi_{a+1}^{j+1}\right) \cdot \xi_{M}\left(\varphi_{a(k)}^{j(k)}\right),
$$

where $\varphi_{a(k)}^{j(k)}$ equals, respectively $\varphi_{a}^{j}, \varphi_{a}^{j+1}, \varphi_{a+1}^{j}, \varphi_{a+1}^{j+1}$, for $k=1, \ldots, 4$. We note that the infinitesimal invariance of $\mathcal{L}_{d}$ can be rewritten as

$$
\left(J_{\mathcal{L}_{d}}^{1}+J_{\mathcal{L}_{d}}^{2}+J_{\mathcal{L}_{d}}^{3}+J_{\mathcal{L}_{d}}^{4}\right)\left(\square_{a}^{j}, \varphi_{a}^{j}, \varphi_{a}^{j+1}, \varphi_{a+1}^{j}, \varphi_{a+1}^{j+1}\right)=0,
$$

for all $j=0, \ldots, N-1$ and $a=0, \ldots, A-1$. This is the statement of the local discrete Noether theorem. To obtain the global discrete Noether theorem, apply formula (46) to variations induced by the group action. Recall that the $G$-action on $J^{1} Y_{d}$ is trivial on the horizontal part and hence there are no horizontal variations. This yields the following result (Marsden et al. [82], Lew et al. [80]).

THEOREM 4. Suppose that the discrete Lagrangian $\mathcal{L}_{d}: J^{1} Y_{d} \rightarrow \mathbb{R}$ is invariant under the action of a Lie group $G$ on $J^{1} Y_{d}$. Suppose that $\varphi_{d}$ is a solution of the discrete covariant Euler-Lagrange equations for $\mathcal{L}_{d}$. Then we have the conservation law

$$
\sum_{\square \in \partial U_{d}^{\prime} \square k ;} \sum_{\square^{(k)} \in \partial U_{d}^{\prime}} J_{\mathcal{L}_{d}}^{k}(\square)=0 .
$$

REMARK 5. Further studies of multisymplectic schemes still need to be developed. For example, error analysis will certainly involve exact generating functionals for multisymplectic field theory; see Vankerschaver et al. [128]. In the case of symplectic time integrators such a study is done via backward error analysis; see Hairer et al. [59]. Other approaches to multisymplectic integrators have been also developed, in, for example, Bridges and Reich [14].

4.2. Multisymplectic integrators for collisions-case I. In this subsection we extend the multisymplectic discretizations, presented in Section 4.1, to the case where the discrete field is subject to a spatial constraint. In the continuous (that is, nondiscretized) situation, such constraints induce singularities 
in the fields. As shown in Section 3.2, the dynamics of these constrained problems can be described in a variational setting by combining the nonsmooth multisymplectic approach developed in Fetecau et al. [41] with the generalized Lagrange multiplier approach for general constrained problems developed, for example, in Rockafellar and Wets [120] and recalled in Section 3.1. We first consider a setting useful to describe the collision of a 1-dimensional elastic bar with a rigid plate.

4.2.1. Discrete geometric setting. Although a more general treatment is possible, we suppose, for simplicity (as in Section 3.2), that $M=\mathbb{R}^{p}$ with the standard inner product, and that the constraint $C$ is the closure of an open subset of $M$. We also assume that $C=(-\psi)^{-1}(P)$, where

$$
\psi=\left(\psi^{1}, \ldots, \psi^{s}\right): \mathbb{R}^{p} \rightarrow \mathbb{R}^{s}
$$

are continuously differentiable functions and

$$
P:=\left\{\left(d^{1}, \ldots, d^{s}\right) \in \mathbb{R}^{s} \mid d^{1} \leqslant 0, \ldots, d^{s} \leqslant 0\right\} .
$$

As in Section 4.1, our approach is illustrated with a spacetime discretization based on a rectangular mesh. Recall that the discrete configuration is a map $\phi_{d}$ : $U_{d} \rightarrow X \times M$, and that the discrete base-space configuration $\phi_{X_{d}}:=\pi_{X Y} \circ \phi_{d}$ : $U_{d} \rightarrow X$ encodes the freedom we allow on the spacetime discretization. We now describe the discrete version of the constraints (23) and (24). The analogue of the constraints (23) are

$$
\phi_{d}\left(U_{d}\right) \subset C,
$$

guaranteeing that the value of the field at each spacetime node is in the constraint subset $C \subset M$. A collision arises at some spacetime location if the corresponding field value belongs to the boundary $\partial C$ of the constraint subset (24). Once a spacetime grid is fixed, such a spacetime location does not coincide with a spacetime node, in general. In our approach, we assume that collisions occur at spatial nodes, but that the corresponding time is determined from the variational principle, thereby extending the approach developed by Fetecau et al. [42]. We now describe the mathematical setting needed to describe this situation. Note that during the dynamics, several collisions can occur at different spatial nodes; however, for simplicity of the exposition, we assume a single collision at time $\tilde{t}=\phi_{X_{d}}^{0}(\widetilde{i})$.

Let $U_{d}=\{0, \ldots, i-1, \widetilde{i}, i, \ldots, N\} \times\{0, \ldots, A\}$. Given fixed space and time steps $\Delta s$ and $\Delta t$, the discrete base-space configuration

$$
\phi_{X_{d}}: U_{d} \rightarrow X=[0, T] \times[0, L]
$$


is defined by

$$
\begin{aligned}
\phi_{X_{d}}(j, a): & =(j \Delta t, a \Delta s)=\left(t^{j}, s_{a}\right) \in X \\
\phi_{X_{d}}(\widetilde{i}, a): & =(\widetilde{t}, a \Delta s)=\left(\widetilde{t}, s_{a}\right) \in X \quad \text { with }(i-1) \Delta t \leqslant \widetilde{t}<i \Delta t .
\end{aligned}
$$

The first equality states that a rectangular spacetime grid is fixed. The second equality gives the time of contact $\tilde{t}=\alpha t^{i}+(1-\alpha) t^{i-1}$ for $\alpha \in[0,1]$, which is the only freedom allowed on the spacetime mesh and it gives rise to horizontal variations.

In addition to the rectangles $\square_{a}^{j}=((j, a),(j+1, a),(j, a+1),(j+1, a+1))$, we also define the rectangles $\square_{a}^{i-1}$ and $\widetilde{\square}_{a}$ by

$$
\begin{aligned}
\square_{a}^{i-1} & :=((i-1, a),(\widetilde{i}, a),(i-1, a+1),(\widetilde{i}, a+1)), \\
\widetilde{\square}_{a} & :=((\widetilde{i}, a),(i, a),(\widetilde{i}, a+1),(i, a+1)) .
\end{aligned}
$$

Let $\varphi_{a}^{j}, \widetilde{\varphi}_{a}$ be the values of the field at the spacetime nodes $(j, a)$ and $(\widetilde{i}, a)$.

We define the analogue of the second part (24) of the constraint by

$$
u \in U_{d}, \quad \phi_{d}(u) \in \partial C \Rightarrow u=(\tilde{i}, a) .
$$

A node $a$ such that $\phi_{d}(\widetilde{i}, a) \in \partial C$ is said to be at contact at time $\tilde{t}$, but note that we attribute the time $\tilde{t}$ to all the other nodes, too.

Define the subset $D_{d}:=\{\widetilde{i}\} \times\{0, \ldots, N\} \subset U_{d}$. Note that it is not the discrete analogue of the singularity subset $D$ in the continuous case. It is rather the spacetime coordinate of all the nodes at the contact time $\tilde{t}$; see Figure 1 .
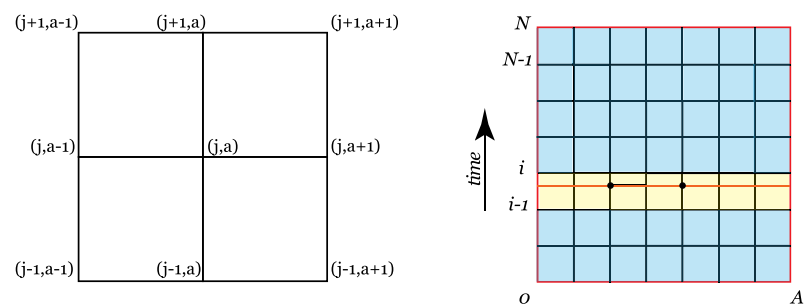

Figure 1. On the left: the rectangles $\square_{a}^{j}, \square_{a}^{j-1}, \square_{a-1}^{j}, \square_{a-1}^{j-1}$. On the right: the discrete parameter space $U_{d}$ and the subset $D_{d}$. The spacetime coordinates of the nodes in contact are designated by $\bullet$.

4.2.2. The discrete action functional. Fix a discrete Lagrangian $\mathcal{L}_{d}: J^{1} Y_{d} \rightarrow \mathbb{R}$, constructed such that the approximation (38) holds. Define, as before, $\mathcal{L}_{a}^{j}:=$ $\mathcal{L}_{d}\left(\square_{a}^{j}, \varphi_{a}^{j}, \varphi_{a}^{j+1}, \varphi_{a+1}^{j}, \varphi_{a+1}^{j+1}\right)$ when $j \neq i-1$. Near the contact time, define 
$\mathcal{L}_{a}^{i-1}:=\mathcal{L}_{d}\left(\square_{a}^{i-1}, \varphi_{a}^{i-1}, \widetilde{\varphi}_{a}, \varphi_{a+1}^{i-1}, \widetilde{\varphi}_{a+1}\right)$ and $\widetilde{\mathcal{L}}_{a}:=\mathcal{L}_{d}\left(\widetilde{\square}_{a}, \widetilde{\varphi}_{a}, \varphi_{a}^{i}, \widetilde{\varphi}_{a+1}, \varphi_{a+1}^{i}\right)$ Thus, the discrete action functional takes the form

$$
\mathfrak{S}_{d}^{n s}\left(\phi_{X_{d}}, \varphi_{d}\right):=\sum_{j=0}^{i-2} \sum_{a=0}^{A-1} \mathcal{L}_{a}^{j}+\sum_{a=0}^{A-1} \mathcal{L}_{a}^{i-1}+\sum_{a=0}^{A-1} \widetilde{\mathcal{L}}_{a}+\sum_{j=i}^{N-1} \sum_{a=0}^{A-1} \mathcal{L}_{a}^{j}
$$

4.2.3. Discrete vertical and horizontal variations. In general, the total derivative of the discrete action function $\mathfrak{S}_{d}^{n s}$ has the form

$$
\begin{aligned}
\mathbf{d} \mathfrak{S}_{d}^{n s} & \left(\phi_{X_{d}}, \varphi_{d}\right) s \cdot\left(\delta \phi_{X_{d}}, \delta \varphi_{d}\right) \\
= & D_{H}^{i n t} \mathfrak{S}_{d}\left(\phi_{X_{d}}, \varphi_{d}\right) \cdot \delta \phi_{X_{d}}^{i n t}+D_{V}^{i n t} \mathfrak{S}_{d}\left(\phi_{X_{d}}, \varphi_{d}\right) \cdot \delta \varphi_{d}^{i n t} \\
& +D_{H}^{\partial} \mathfrak{S}_{d}\left(\phi_{X_{d}}, \varphi_{d}\right) \cdot \delta \phi_{X_{d}}^{\partial}+D_{V}^{\partial} \mathfrak{S}_{d}\left(\phi_{X_{d}}, \varphi_{d}\right) \cdot \delta \varphi_{d}^{\partial} \\
& +D_{H}^{\sim, i n t} \mathfrak{S}_{d}\left(\phi_{X_{d}}, \varphi_{d}\right) \cdot \delta \widetilde{\phi}_{X_{d}}^{i n t}+D_{V}^{\sim, i n t} \mathfrak{S}_{d}\left(\phi_{X_{d}}, \varphi_{d}\right) \cdot \delta \widetilde{\varphi}_{d}^{i n t} \\
& +D_{H}^{\sim, \partial} \mathfrak{S}_{d}\left(\phi_{X_{d}}, \varphi_{d}\right) \cdot \delta \widetilde{\phi}_{X_{d}}^{\partial}+D_{V}^{\sim, \partial} \mathfrak{S}_{d}\left(\phi_{X_{d}}, \varphi_{d}\right) \cdot \delta \widetilde{\varphi}_{d}^{\partial} .
\end{aligned}
$$

Here $\delta \phi_{X_{d}}^{\text {int }}$, respectively $\delta \phi_{X_{d}}^{\partial}$, denote the horizontal variations away from impact, at the interior, respectively, the boundary of the domain; $\delta \varphi_{d}^{\text {int }}$, respectively $\delta \varphi_{d}^{\partial}$, denote the vertical variations away from the contact time, at the interior, respectively, the boundary of the domain. The notations $\delta \widetilde{\phi}_{X_{d}}^{\partial}, \delta \widetilde{\phi}_{X_{d}}^{i n t}, \delta \widetilde{\varphi}_{d}^{i n t}, \delta \widetilde{\varphi}_{d}^{\partial}$ have the same meaning at the contact time. The notations on the various derivatives correspond to these variations. From our choice (53) of discrete basespace configuration, the variations $\delta \phi_{X_{d}}^{i n t}$ and $\delta \phi_{X_{d}}^{\partial}$ do not appear in the formula for $\mathbf{d} \mathfrak{S}_{d}^{n s}$, as opposed to (40), where the discrete base space is defined by (36).

Taking the variation of each of the terms in (55), we get

$$
\begin{aligned}
\mathbf{d} \mathfrak{S}_{d}^{n s} & \left(\phi_{X_{d}}, \varphi_{d}\right) \cdot\left(\delta \phi_{X_{d}}, \delta \varphi_{d}\right) \\
= & \sum_{j=1}^{i-1} \sum_{a=1}^{A-1}\left(D_{1} \mathcal{L}_{a}^{j}+D_{2} \mathcal{L}_{a}^{j-1}+D_{3} \mathcal{L}_{a-1}^{j}+D_{4} \mathcal{L}_{a-1}^{j-1}\right) \cdot \delta \varphi_{a}^{j} \\
& +\sum_{j=i+1}^{N-1} \sum_{a=1}^{A-1}\left(D_{1} \mathcal{L}_{a}^{j}+D_{2} \mathcal{L}_{a}^{j-1}+D_{3} \mathcal{L}_{a-1}^{j}+D_{4} \mathcal{L}_{a-1}^{j-1}\right) \cdot \delta \varphi_{a}^{j} \\
& +\sum_{a=1}^{A-1}\left(D_{1} \mathcal{L}_{a}^{i}+D_{2} \widetilde{\mathcal{L}}_{a}+D_{3} \mathcal{L}_{a-1}^{i}+D_{4} \widetilde{\mathcal{L}}_{a-1}\right) \cdot \delta \varphi_{a}^{i} \\
& +\sum_{\square \in U_{d}^{\square}} \sum_{\square\left(\square^{(k)} \in \partial U_{d} \cup D_{d}\right.} D_{k} \mathcal{L}(\square) \cdot \delta \varphi_{a}^{j}+\sum_{a=0}^{A-1}\left(D_{\tilde{t}} \mathcal{L}_{a}^{i-1}+D_{\tilde{t}} \widetilde{\mathcal{L}}_{a}\right) \delta \widetilde{t},
\end{aligned}
$$

where, in the fourth line, the index $(a, j)$ in $\delta \varphi_{a}^{j}$ corresponds to the coordinate of the node $k=1,2,3,4$ (see (37)). The first and second lines correspond to interior 
vertical variations, the third line to vertical variations at the boundary and along the contact time, the last line to horizontal variations.

Note that $D_{\tilde{t}} \mathcal{L}_{a}^{i-1}$ denotes the derivative relative to the variables $\tilde{t}$ which appear through the dependence of $\mathcal{L}_{d}$ on $\square_{a}^{i-1}$ identified with $\left(s_{a}, t^{i-1}, s_{a+1}, \tilde{t}\right)$. Similarly, $D_{\tilde{t}} \widetilde{\mathcal{L}}_{a}$ denotes the derivative relative to $\widetilde{t}$, where $\widetilde{\square}_{a}$ is identified with $\left(s_{a}, \tilde{t}, s_{a+1}, t^{i}\right)$. The horizontal variation can equivalently be taken with respect to $\alpha$, when writing $\tilde{t}=t^{i-1}+\alpha \Delta t$ for $\alpha \in[0,1]$, in which case we have $\delta \tilde{t}=\Delta t \delta \alpha$. If the Lagrangian does not depend explicitly on time, then $\mathcal{L}_{d}$ depends on $\tilde{t}$ only through the time steps mentioned above, that is, $\tilde{t}-t^{i-1}=\alpha \Delta t$ and $t^{i}-\tilde{t}=(1-\alpha) \Delta t$. In this case, the horizontal variations (the last summand in (57)) can be equivalently written as $\sum_{a=0}^{A-1}\left(D_{h} \mathcal{L}_{a}^{i-1}-D_{h} \widetilde{\mathcal{L}}_{a}\right) \delta \widetilde{t}$, where $D_{h}$ denotes the derivative relative to the time step.

In terms of the discrete Cartan form defined in (44), the sum of the third and fourth terms in (57) can be rewritten as

$$
\begin{aligned}
& \sum_{\square \in U_{d}^{\square}} \sum_{k ; \square(k) \in D_{d}}\left[\left(j^{1} \varphi_{d}\right)^{*} \mathbf{i}_{j^{1} V_{d}} \Theta_{\mathcal{L}_{d}}^{k}\right](\square) \\
& \quad=\sum_{\square \in U_{d}^{\square}} \sum_{k ; \square^{(k)} \in D_{d}} D_{k} \mathcal{L}(\square) \cdot \delta \varphi_{a}^{j}+\sum_{a=0}^{A-1}\left(D_{\tilde{t}} \mathcal{L}_{a}^{i-1}+D_{\tilde{t}} \widetilde{\mathcal{L}}_{a}\right) \delta \tilde{t}
\end{aligned}
$$

and is the discrete analogue of the jump term in (9). The discrete analogue of the boundary term in (9) is

$$
\sum_{\square \in U_{d}^{\square}} \sum_{k ; \square(k) \in \partial U_{d}}\left[\left(j^{1} \varphi_{d}\right)^{*} \mathbf{i}_{j^{1} V_{d}} \Theta_{\mathcal{L}_{d}}^{k}\right](\square) .
$$

Note that the terms associated to $\delta \widetilde{\varphi}_{0}$ and $\delta \widetilde{\varphi}_{A}$ belong to both (58) and (59). This is due to the fact that $\widetilde{i}, 0), \widetilde{i}, A) \in \partial U_{d} \cap D_{d}$.

Below, assume that the configuration is known at $t^{0}$ and $t^{N}$, that is, $\delta \varphi_{a}^{0}=0$ and $\delta \varphi_{a}^{N}=0$, for all $a=0, \ldots, A$. On the spatial boundary we use zero-traction boundary conditions, so that the variations $\delta \varphi_{0}^{j}$ and $\delta \varphi_{A}^{j}$ are arbitrary for all $j=1, \ldots, N-1$ and $j=\widetilde{i}$.

4.2.4. Generalized Lagrange multiplier approach. We now write the necessary conditions on $\phi_{d}$ to be a critical point of $\mathfrak{S}_{d}^{n s}$ with respect to the constraints (52) and (54). From Theorem 1, a necessary condition is given by

$$
-\left(\frac{\delta \mathfrak{S}_{d}^{n s}}{\delta \phi_{d}}\right)_{a}^{j} \in N_{C}\left(\varphi_{a}^{j}\right), \quad \forall(j, a) \in U_{d} .
$$


Recall that the normal cone is

$$
N_{C}\left(\varphi_{a}^{j}\right)=\left\{\lambda_{1}(j, a) \nabla \psi^{1}\left(\varphi_{a}^{j}\right)+\cdots+\lambda_{s}(j, a) \nabla \psi^{s}\left(\varphi_{a}^{j}\right) \mid \lambda(j, a) \in N_{P}\left(-\psi\left(\varphi_{a}^{j}\right)\right)\right\} .
$$

We thus obtain the following cases.

(i) If $(j, a) \in U_{d} \backslash\left(\partial U_{d} \cup D_{d}\right)=\{1, \ldots, N-1\} \times\{1, \ldots, A-1\}$, then from (52) and (54) we have $\varphi_{a}^{j} \in \operatorname{int}(C)$. Since we assumed that $C$ is the closure of an open subset of $M$, the normal cone to $C$ at $\varphi_{a}^{j}$ in $M$ reduces to zero. In this case, using (57), the condition (60) yields the discrete covariant Euler-Lagrange equations

$$
D_{1} \mathcal{L}_{a}^{j}+D_{2} \mathcal{L}_{a}^{j-1}+D_{3} \mathcal{L}_{a-1}^{j}+D_{4} \mathcal{L}_{a-1}^{j-1}=0, \quad \forall(j, a) \in U_{d} \backslash\left(\partial U_{d} \cup D_{d}\right) .
$$

(ii) If $(j, a) \in D_{d}=\{\tilde{i}\} \times\{0, \ldots, A\}$, then from (52) and (54) we can have $\varphi_{a}^{j}=\widetilde{\varphi}_{a} \in \partial C$ or $\varphi_{a}^{j}=\widetilde{\varphi}_{a} \in \operatorname{int}(C)$. Under the hypothesis (21) on $\psi$ and $\lambda$, using (57), the vertical part of condition (60) yields

$$
\left.D_{1} \widetilde{\mathcal{L}}_{a}+D_{2} \mathcal{L}_{a}^{i-1}+D_{3} \widetilde{\mathcal{L}}_{a-1}+D_{4} \mathcal{L}_{a-1}^{i-1}=\lambda \widetilde{i}, a\right) \cdot \nabla \psi\left(\widetilde{\varphi}_{a}\right)
$$

where $\lambda(\widetilde{i}, a) \in N_{P}\left(-\psi\left(\widetilde{\varphi}_{a}\right)\right)$. We note that $\lambda(\widetilde{i}, a)$ is zero if $a \in\{0, \ldots, N\}$ is such that $\widetilde{\varphi}_{a} \in \operatorname{int}(C)$, that is, if the node $a$ is not in contact at time $\widetilde{t}$.

The horizontal part of (60) yields energy conservation during the impact

$$
\sum_{a=0}^{A-1}\left(D_{h} \mathcal{L}_{a}^{i-1}-D_{h} \widetilde{\mathcal{L}}_{a}\right)=0
$$

(iii) We now consider the case $(j, a) \in \partial U_{d} \backslash D_{d}$. If $j=0$ or $j=N$, there are no corresponding equations by our choice of boundary conditions since $\delta \varphi_{a}^{0}=0$ and $\delta \varphi_{a}^{N}=0$, for all $a=0, \ldots, A$. If $a=0$ or $a=A$, we get the zero-traction boundary conditions, for all $j=1, \ldots, N-1$ :

$$
D_{1} \mathcal{L}_{0}^{j}+D_{2} \mathcal{L}_{0}^{j-1}=0, \quad D_{3} \mathcal{L}_{A-1}^{j}+D_{4} \mathcal{L}_{A-1}^{j-1}=0
$$

Note that if $a=0$ or $a=A$ in (62), then it takes the specific form $D_{1} \widetilde{\mathcal{L}}_{0}+D_{2} \mathcal{L}_{0}^{i-1}=\lambda(\widetilde{i}, 0) \cdot \nabla \psi\left(\widetilde{\varphi}_{0}\right), \quad D_{3} \widetilde{\mathcal{L}}_{A-1}+D_{4} \mathcal{L}_{A-1}^{i-1}=\lambda(\widetilde{i}, A) \cdot \nabla \psi\left(\widetilde{\varphi}_{A}\right)$.

So, if $a=0$, or $a=A$ is not in contact at time $\tilde{t}$, the Lagrange multiplier vanishes and these equations become the zero-traction boundary conditions at time $\tilde{t}$, thereby completing (63) at the contact time.

These results are summarized in the following theorem. 
THEOREM 5. Assume the hypotheses of Theorem 3 on the bundle $\pi_{X Y}: Y \rightarrow X$, the constraint $C$, and the Lagrange multiplier $\lambda$. Consider the discrete setting of Section 4.2.1, with discrete base-space configuration (53). Consider a discrete Lagrangian density $\mathcal{L}_{d}: J^{1} Y_{d} \rightarrow \mathbb{R}$ and the associated discrete action functional $\mathfrak{S}_{d}^{n s}$ defined in (55). If $\phi_{d}=\left(\phi_{X_{d}}, \varphi_{d}\right)$ is a critical point of $\mathfrak{S}_{d}^{n s}$ relative to the constraints (52) and (54), then:

- Away from the contact time, the fields $\phi_{X_{d}}$ and $\varphi_{d}$ satisfy the discrete covariant Euler-Lagrange equations

$$
D_{1} \mathcal{L}_{a}^{j}+D_{2} \mathcal{L}_{a}^{j-1}+D_{3} \mathcal{L}_{a-1}^{j}+D_{4} \mathcal{L}_{a-1}^{j-1}=0, \quad \forall(j, a) \in U_{d} \backslash\left(\partial U_{d} \cup D_{d}\right) .
$$

- At the contact time, the fields $\phi_{X_{d}}$ and $\varphi_{d}$ verify the following conditions:

(a) the energy conservation at the contact time:

$$
\sum_{a=0}^{A-1}\left(D_{h} \mathcal{L}_{a}^{i-1}-D_{h} \widetilde{\mathcal{L}}_{a}\right)=0
$$

(b) the vertical discrete jump condition for $\lambda(\widetilde{i}, a) \in N_{P}\left(-\psi\left(\widetilde{\varphi}_{a}\right)\right)$ :

$$
D_{1} \widetilde{\mathcal{L}}_{a}+D_{2} \mathcal{L}_{a}^{i-1}+D_{3} \widetilde{\mathcal{L}}_{a-1}+D_{4} \mathcal{L}_{a-1}^{i-1}=\lambda(\widetilde{i}, a) \cdot \nabla \psi\left(\widetilde{\varphi}_{a}\right), \quad \forall a \neq 0, A .
$$

Note that $\lambda(\widetilde{i}, a)=0$ if the node a is not in contact at time $\widetilde{t}$.

- At the spatial boundary, away from the contact time, the field $\varphi_{d}$ verifies the zero-traction boundary conditions

$$
D_{1} \mathcal{L}_{0}^{j}+D_{2} \mathcal{L}_{0}^{j-1}=0, \quad D_{3} \mathcal{L}_{A-1}^{j}+D_{4} \mathcal{L}_{A-1}^{j-1}=0, \quad \forall j=1, \ldots, N-1 .
$$

REMARK 6 . Note that if the contact happens at the extremity $a=0$, respectively $a=A$, then the zero-traction boundary condition is replaced by the expression

$$
\begin{aligned}
& D_{1} \widetilde{\mathcal{L}}_{0}+D_{2} \mathcal{L}_{0}^{i-1}=\lambda(\widetilde{i}, 0) \cdot \nabla \psi\left(\widetilde{\varphi}_{0}\right), \quad \text { respectively } \\
& D_{3} \widetilde{\mathcal{L}}_{A-1}+D_{4} \mathcal{L}_{A-1}^{i-1}=\lambda(\widetilde{i}, A) \cdot \nabla \psi\left(\widetilde{\varphi}_{A}\right) .
\end{aligned}
$$

REMARK 7. We give the details of the computation of the contact time $\tilde{t}$. Let $t^{j+1}$ be the first value for which there exists an $a \in\{0, \ldots, A\}$ such that $-\psi\left(\varphi_{a}^{j+1}\right) \notin P$ (see (28) and (29)). Then we search for $\left.\tilde{t}=t^{j}+\alpha \Delta t \in\right] t^{j}, t^{j+1}[$ such that $-\psi\left(\widetilde{\varphi}_{a}\right) \in \partial P$ and either (64) holds if $a \in\{1, \ldots, A-1\}$ or (67) holds if $a \in\{0, A\}$. This rule is applied to the examples in Section 5. See also Demoures et al. [30], where we give a detailed algorithm in two concrete examples. 
4.2.5. Discrete multisymplectic form formula. Consider the discrete action functional $\mathfrak{S}_{d}^{n s}$ defined in (55). With the help of the discrete Cartan forms defined in (44), the derivative of $\mathfrak{S}_{d}^{n s}$ computed in (57) is

$$
\mathbf{d} \mathfrak{S}_{d}^{n s}\left(\phi_{d}\right) \cdot V_{d}=\sum_{\square \in U_{d}^{\square}} \sum_{k \in \square}\left[\left(j^{1} \phi_{d}\right)^{*}\left(\mathbf{i}_{j^{1} V_{d}} \Theta_{\mathcal{L}_{d}}^{k}\right)\right](\square),
$$

where $\phi_{d}=\left(\phi_{X_{d}}, \varphi_{d}\right)$. This derivative is computed on the space of all basespace configurations of the form (53) and for all possible discrete fields $\varphi_{d}$. In this formula, we did not impose any constraints on the discrete fields. With this particular choice of base-space configurations, the first terms in the discrete Cartan forms (44) only appear when $j=\tilde{i}$.

Choose a subdomain $U_{d}^{\prime}$ of $U_{d}$ and restrict the discrete action functional $\mathfrak{S}_{d}^{n s}$ to discrete configurations restricted to $U_{d}^{\prime}$, which we denote by $\mathfrak{S}_{d}^{\prime n s}$. The derivative of $\mathfrak{S}_{d}^{\prime \prime n}$ has the same expression as (68), with $U_{d}$ replaced by $U_{d}^{\prime}$. Note that if $\phi_{d}$ verifies the conditions of Theorem 5 then the derivative of $\mathfrak{S}_{d}^{\prime}{ }^{n s}$ reads

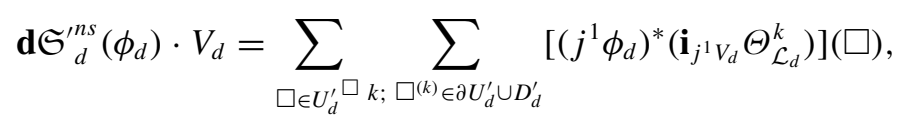

where $D_{d}^{\prime}=U_{d}^{\prime \square} \cap D_{d}$. Note that to obtain this formula we only used a subset of the conditions found in Theorem 5, namely, we only used the discrete EulerLagrange equations associated to the nodes $(j, a) \in U_{d} \backslash\left(\partial U_{d} \cup D_{d}\right)$, that is, equations (64).

Taking one exterior derivative and evaluating it on first variations $V_{d}, W_{d}$ of a solution $\phi_{d}=\left(\phi_{X_{d}}, \varphi_{d}\right)$ of (64), we get the discrete multisymplectic form formula

$$
\sum_{\square \in U_{d}^{\prime} \square} \sum_{k ; \square(k) \in \partial U_{d}^{\prime} \cup D_{d}^{\prime}}\left[\left(j^{1} \phi_{d}\right)^{*}\left(\mathbf{i}_{j^{1} V_{d}} \mathbf{i}_{j^{1} W_{d}} \Omega_{\mathcal{L}_{d}}^{k}\right)\right](\square)=0,
$$

where we recall that $\Omega_{\mathcal{L}_{d}}^{k}, k=1, \ldots, 4$ are the Cartan 2-forms on $J^{1} Y_{d}$ defined by $\Omega_{\mathcal{L}_{d}}^{k}:=-\mathbf{d} \Theta_{\mathcal{L}_{d}}^{k}$. We also recall that the expression in (70) depends on both the horizontal and vertical components of $V_{d}$ and $W_{d}$. Formula (70) is the discrete version of (20).

4.2.6. Discrete momentum maps and discrete Noether theorem. We now extend the discrete Noether theorem (as recalled in Theorem 4) to the case with constraints. As before, we only consider vertical symmetries. Let $\Phi: G \times M \rightarrow M$ be a left action of a Lie group $G$ on $M$ and assume that the discrete Lagrangian $\mathcal{L}_{d}$ is invariant under the $G$-action on $J^{1} Y_{d}$. Recall that the discrete momentum maps are defined by

$$
J_{\mathcal{L}_{d}}^{k}: J^{1} Y_{d} \rightarrow \mathfrak{g}^{*}, \quad\left\langle J_{\mathcal{L}_{d}}^{k}, \xi\right\rangle:=\mathbf{i}_{\xi_{J^{1} Y_{d}}} \Theta_{\mathcal{L}}^{k}, \quad \xi \in \mathfrak{g}, k=1, \ldots, 4
$$


To obtain the global discrete Noether theorem, we proceed exactly as in the continuous case, namely, we apply formula (57) for the variations (restricted to a subset $U_{d}^{\prime}$ ) along the group action, and make use of the necessary conditions for a critical point. Denoting $D_{d}^{\prime}:=D_{d} \cap U_{d}^{\prime}$, if $\phi_{d}$ satisfies the necessary conditions of Theorem 5, then

$$
\sum_{\square \in U_{d}^{\prime} \square} \sum_{k ; \square(k) \in \partial U_{d}^{\prime} \cup D_{d}^{\prime}}\left[\left(j^{1} \varphi_{d}\right)^{*} \mathbf{i}_{j^{1} \xi_{Y_{d}}} \Theta_{\mathcal{L}_{d}}^{k}\right](\square)=0, \quad \forall \xi \in \mathfrak{g} .
$$

By using the definition (71) of the discrete momentum maps, we get the following result that generalizes Theorem 4 .

THEOREM 6. Suppose that the discrete Lagrangian $\mathcal{L}_{d}: J^{1} Y_{d} \rightarrow \mathbb{R}$ is invariant under the action of a Lie group $G$ on $J^{1} Y_{d}$. Suppose that $\phi_{d}$ verifies the necessary conditions of Theorem 5. Consider a subdomain $U_{d}^{\prime} \subset U_{d}$ and define $D_{d}^{\prime}:=D_{d} \cap U_{d}^{\prime}$. Then $\phi_{d}$ verifies the conservation law

$$
\sum_{\square \in U_{d}^{\prime} \square} \sum_{k ; \square(k) \in \partial U_{d}^{\prime} \cup D_{d}^{\prime}} J_{\mathcal{L}_{d}}^{k}(\square)=0 .
$$

To obtain this conservation law we only used the first condition of Theorem 5, that is, the discrete covariant Euler-Lagrange equations away from contact time. Using condition (b) in Theorem 5, we can rewrite the terms in (72) for which $\square^{(k)} \in D_{d}^{\prime}$, in terms of the Lagrange multipliers (that turn out to vanish for all nodes $a$ that are not in contact at the contact time). Note also that if $U_{d}^{\prime} \cap D_{d}=\varnothing$, (72) recovers the Noether conservation law of Theorem 4 in absence of constraints. Note, finally, that this theorem generalizes, to the multisymplectic setting, the Noether theorem for variational collision integrators in Lagrangian mechanics developed in Fetecau et al. [42, Theorem 3.2].

4.3. Multisymplectic integrators for collisions-case II. We extend the framework of Section 4.2 to treat the collision of two 1-dimensional bodies.

4.3.1. Discrete geometric setting. For two bodies, the discrete parameter space is

$$
U_{d}=\{0, \ldots, i-1, \widetilde{i}, i, \ldots, N\} \times(\{0, \ldots, A\} \sqcup\{0, \ldots, B\}) \ni(j, a, b) .
$$

The associated rectangles are denoted $\square_{a}^{j}, \widetilde{\square}_{a}$ and $\square_{b}^{j}, \widetilde{\square}_{b}$. Define the subset

$$
D_{d}:=\{\widetilde{i}\} \times(\{0, \ldots, A\} \sqcup\{0, \ldots, B\}) .
$$

A discrete configuration is given, as before, by a map $\phi_{d}: U_{d} \rightarrow X \times M$, where $X=[0, T] \times\left(\left[0, L_{1}\right] \sqcup\left[0, L_{2}\right]\right)$. Given fixed space and time steps $\Delta s$ and $\Delta t$, 

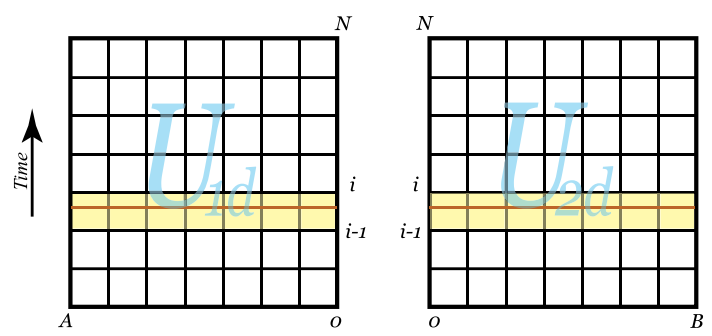

Figure 2. Discrete spacetime subdomains $U_{1 d}, U_{2 d}$, and the subsets $D_{1 d}, D_{2 d}$.

the discrete base-space configuration $\phi_{X_{d}}: U_{d} \rightarrow X$ is defined by

$$
\begin{aligned}
\phi_{X_{d}}(j, a, b) & =(j \Delta t, a \Delta s, b \Delta s)=\left(t^{j}, s_{a}, s_{b}\right) \in X \\
\phi_{X_{d}}(\widetilde{i}, a, b): & =(\widetilde{t}, a \Delta s, b \Delta s) \in X \quad \text { with }(i-1) \Delta t \leqslant \widetilde{t}<i \Delta t .
\end{aligned}
$$

The first equality means that a rectangular spacetime grid is fixed for each body. The second equality gives the time of contact of the two bodies, which has to be determined. As before, it can also be equivalently given by a parameter $\alpha \in[0,1]$ such that $\tilde{t}=\alpha t^{i-1}+(1-\alpha) t^{i}$.

It is convenient to also define the discrete parameter spaces

$$
U_{1 d}=\{0, \ldots, N\} \times\{0, \ldots, A\}, \quad U_{2 d}=\{0, \ldots, N\} \times\{0, \ldots, B\},
$$

and the subsets $D_{1 d}=\{\widetilde{i}\} \times\{0, \ldots, A\}, D_{2 d}=\{\widetilde{i}\} \times\{0, \ldots, B\}$. We have $U_{d}=U_{1 d} \cup U_{2 d}$ and $D_{d}=D_{1 d} \cup D_{2 d}$; see Figure 2. To treat the problem of the collision of two bars, the constraint on the discrete field is induced by a constraint subset $C \subset M \times M$. The constraint on the field is

$$
\begin{array}{cl}
\left(\varphi_{a}^{j}, \varphi_{b}^{j}\right) \subset C & \text { for all }(j, a, b) \in U_{d}, \quad \text { and } \\
\left(\varphi_{a}^{j}, \varphi_{b}^{j}\right) \subset \partial C & \Rightarrow(j, a, b) \in D_{d}, \quad \text { that is, } j=\tilde{i} .
\end{array}
$$

4.3.2. The discrete action functional. Fix a discrete Lagrangian $\mathcal{L}_{d}: J^{1} Y_{d} \rightarrow \mathbb{R}$, such that the approximation (38) holds. Define, exactly as before, $\mathcal{L}_{a}^{j}, \mathcal{L}_{b}^{j}, \mathcal{L}_{a}^{i-1}$, $\mathcal{L}_{b}^{i-1}, \widetilde{\mathcal{L}}_{a}$, and $\widetilde{\mathcal{L}}_{b}$. The discrete action functional is

$$
\begin{aligned}
\mathfrak{S}_{d}^{n s}\left(\phi_{1 d}, \phi_{2 d}\right)= & \sum_{j=0}^{i-2}\left(\sum_{a=0}^{A-1} \mathcal{L}_{a}^{j}+\sum_{b=0}^{B-1} \mathcal{L}_{b}^{j}\right)+\left(\sum_{a=0}^{A-1} \mathcal{L}_{a}^{i-1}+\sum_{b=0}^{B-1} \mathcal{L}_{b}^{i-1}\right) \\
& +\left(\sum_{a=0}^{A-1} \widetilde{\mathcal{L}}_{a}+\sum_{b=0}^{B-1} \widetilde{\mathcal{L}}_{b}\right)+\sum_{j=i}^{N-1}\left(\sum_{a=0}^{A-1} \mathcal{L}_{a}^{j}+\sum_{b=0}^{B-1} \mathcal{L}_{b}^{j}\right) .
\end{aligned}
$$


4.3.3. Discrete vertical and horizontal variations. The abstract expression of the derivative of the discrete action functional has the same form as before, namely (56). Similar computations as in Section 4.2.3 yield

$$
\begin{aligned}
& \mathbf{d S}_{d}^{n s}\left(\phi_{X_{d}}, \varphi_{d}\right) \cdot\left(\delta \phi_{X_{d}}, \delta \varphi_{d}\right) \\
& =\sum_{j=1}^{i-1} \sum_{\gamma \in\{a, b\}} \sum_{\gamma=1}^{\Gamma-1}\left(D_{1} \mathcal{L}_{\gamma}^{j}+D_{2} \mathcal{L}_{\gamma}^{j-1}+D_{3} \mathcal{L}_{\gamma-1}^{j}+D_{4} \mathcal{L}_{\gamma-1}^{j-1}\right) \cdot \delta \varphi_{\gamma}^{j} \\
& +\sum_{j=i+1}^{N-1} \sum_{\gamma \in\{a, b\}} \sum_{\gamma=1}^{\Gamma-1}\left(D_{1} \mathcal{L}_{\gamma}^{j}+D_{2} \mathcal{L}_{\gamma}^{j-1}+D_{3} \mathcal{L}_{\gamma-1}^{j}+D_{4} \mathcal{L}_{\gamma-1}^{j-1}\right) \cdot \delta \varphi_{\gamma}^{j} \\
& +\sum_{\gamma \in\{a, b\}} \sum_{\gamma=1}^{\Gamma-1}\left(D_{1} \mathcal{L}_{\gamma}^{i}+D_{2} \widetilde{\mathcal{L}}_{\gamma}+D_{3} \mathcal{L}_{\gamma-1}^{i}+D_{4} \widetilde{\mathcal{L}}_{\gamma-1}\right) \cdot \delta \varphi_{\gamma}^{i} \\
& +\sum_{\square \in \partial U_{1 d}^{\square}}\left(\sum_{k ; \square(k) \in \partial U_{1 d} \cup D_{1 d}} D_{k} \mathcal{L}(\square) \cdot \delta \varphi_{a}^{j}\right) \\
& +\sum_{\square \in \partial U_{2 d}^{\square}}\left(\sum_{k ; \square(k) \in \partial U_{2 d} \cup D_{2 d}} D_{k} \mathcal{L}(\square) \cdot \delta \varphi_{b}^{j}\right) \\
& +\left(\sum_{a=0}^{A-1}\left(D_{\tilde{t}} \mathcal{L}_{a}^{i-1}+D_{\tilde{t}} \widetilde{\mathcal{L}}_{a}\right)+\sum_{b=0}^{B-1}\left(D_{\tilde{t}} \mathcal{L}_{b}^{i-1}+D_{\tilde{t}} \widetilde{\mathcal{L}}_{b}\right)\right) \delta \tilde{t},
\end{aligned}
$$

where, in the first, second, and third line $\Gamma=A$ if $\gamma=a$ and $\Gamma=B$ if $\gamma=b$, whereas in the fourth line, the index $(\gamma, j)$ in $\delta \varphi_{\gamma}^{j}$ corresponds to the coordinate of the node $k$ (see (37)).

4.3.4. Generalized Lagrange multiplier approach. We now determine the necessary conditions on $\phi_{d}$ to be a critical point of $\mathfrak{S}_{d}^{n s}$ with respect to the constraints (74) and (75). From Theorem 1, a necessary condition is given by

$$
-\left(\frac{\delta \mathfrak{S}_{d}^{n s}}{\delta \phi_{d}}\right)_{a, b}^{j} \in N_{C}\left(\varphi_{a}^{j}, \varphi_{b}^{j}\right), \quad \forall(j, a) \in U_{d} .
$$

Repeating the same arguments as in Section 4.2.4, we get the following result.

THEOREM 7. Assume the hypotheses of Theorem 3 on the bundle $\pi_{X Y}: Y \rightarrow X$, the constraint $C$, and the Lagrange multiplier $\lambda$. Consider the discrete setting of Section 4.2.1, with discrete base-space configuration (73). Consider a discrete Lagrangian density $\mathcal{L}_{d}: J^{1} Y_{d} \rightarrow \mathbb{R}$ and the associated discrete action 
functional $\mathfrak{S}_{d}^{n s}$ defined in (55). If $\phi_{d}=\left(\phi_{X_{d}}, \varphi_{d}\right)$ is a critical point of $\mathfrak{S}_{d}^{n s}$ relative to the constraints (52) and (54), then:

- Away from the contact time, the fields $\phi_{X_{d}}$ and $\varphi_{d}$ satisfy the discrete covariant Euler-Lagrange equations

$$
\begin{aligned}
& D_{1} \mathcal{L}_{a}^{j}+D_{2} \mathcal{L}_{a}^{j-1}+D_{3} \mathcal{L}_{a-1}^{j}+D_{4} \mathcal{L}_{a-1}^{j-1}=0, \quad \forall(j, a) \in U_{1 d} \backslash\left(\partial U_{1 d} \cup D_{1 d}\right) \\
& D_{1} \mathcal{L}_{b}^{j}+D_{2} \mathcal{L}_{b}^{j-1}+D_{3} \mathcal{L}_{b-1}^{j}+D_{4} \mathcal{L}_{b-1}^{j-1}=0, \quad \forall(j, b) \in U_{2 d} \backslash\left(\partial U_{2 d} \cup D_{2 d}\right) .
\end{aligned}
$$

- At the contact time, the fields $\phi_{X_{d}}$ and $\varphi_{d}$ verify the following conditions:

(a) the energy conservation at the contact time

$$
\sum_{a=0}^{A-1}\left(D_{h} \mathcal{L}_{a}^{i-1}-D_{h} \widetilde{\mathcal{L}}_{a}\right)+\sum_{b=0}^{B-1}\left(D_{h} \mathcal{L}_{b}^{i-1}-D_{h} \widetilde{\mathcal{L}}_{b}\right)=0
$$

(b) the vertical discrete jump condition

$$
\left(\begin{array}{c}
D_{1} \widetilde{\mathcal{L}}_{a}+D_{2} \mathcal{L}_{a}^{i-1}+D_{3} \widetilde{\mathcal{L}}_{a-1}+D_{4} \mathcal{L}_{a-1}^{i-1} \\
D_{1} \widetilde{\mathcal{L}}_{b}+D_{2} \mathcal{L}_{b}^{i-1}+D_{3} \widetilde{\mathcal{L}}_{b-1}+D_{4} \mathcal{L}_{b-1}^{i-1}
\end{array}\right)=\lambda(\widetilde{i}, a, b) \cdot \nabla \psi\left(\widetilde{\varphi}_{a}, \widetilde{\varphi}_{b}\right),
$$

for all $a=0, \ldots, A$ and $b=0, \ldots, B$, where $\lambda(\tilde{i}, a, b) \in N_{P}\left(-\psi\left(\widetilde{\varphi}_{a}, \widetilde{\varphi}_{b}\right)\right)$. We recall that $\lambda(\widetilde{i}, a, b)$ is zero if the nodes $a$ and $b$ are not in contact at time $\tilde{t}$.

- At the spatial boundary, away from the contact time, the field $\varphi_{d}$ verifies the zero-traction boundary conditions

$$
\begin{array}{ll}
D_{1} \mathcal{L}_{a=0}^{j}+D_{2} \mathcal{L}_{a=0}^{j-1}=0, & D_{3} \mathcal{L}_{A-1}^{j}+D_{4} \mathcal{L}_{A-1}^{j-1}=0, \\
D_{1} \mathcal{L}_{b=0}^{j}+D_{2} \mathcal{L}_{b=0}^{j-1}=0, & D_{3} \mathcal{L}_{B-1}^{j}+D_{4} \mathcal{L}_{B-1}^{j-1}=0,
\end{array} \quad \forall j=1, \ldots, N-1 .
$$

As explained in Remark 6, if the contact happens at a boundary, then one needs to modify the associated zero-traction boundary condition.

4.3.5. Discrete multisymplectic form formula. To obtain the discrete multisymplectic form formula, we proceed, exactly as in Section 4.2.5, with $U_{d}=U_{1 d} \cup U_{2 d}$ and $D_{d}=D_{1 d} \cup D_{2 d}$. Given a subdomain $U_{d}^{\prime} \subset U_{d}$, if $\phi_{d}$ verifies the condition of Theorem 7 , then the derivative of the action functional has the expression (69), where only the conditions (78) were used. We thus get the same formula as earlier, that is, (70), where $V_{d}, W_{d}$ are now first variations of (78) and $D_{d}^{\prime}=U_{d}^{\prime} \cap D_{d}$. 


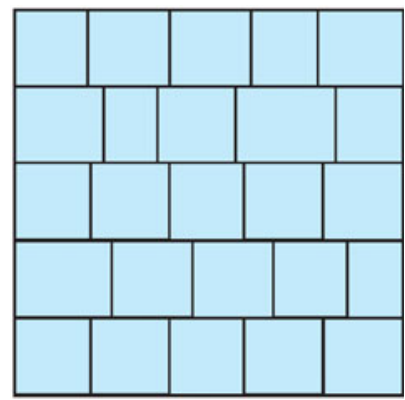

Figure 3. An example of discrete spacetime with time-dependent spatial discretization.

\subsubsection{Discrete momentum map and discrete Noether theorem. Using (77) and} proceeding as in Section 4.2.6, the discrete Noether theorem takes exactly the form in Theorem 6. In this case, note, however, that $\partial U_{d}^{\prime} \cup D_{d}^{\prime}=\partial U_{1 d}^{\prime} \cup D_{1 d}^{\prime} \cup$ $\partial U_{2 d}^{\prime} \cup D_{2 d}^{\prime}$. If $U_{d}^{\prime} \subset U_{1 d}$, then this Noether theorem reduces to a Noether theorem for the motion of the first body. If, furthermore, $U_{d}^{\prime} \cap D_{1 d}=\varnothing$, we recover Theorem 4.

REMARK 8. In Theorems 5 and 7 we have chosen, for simplicity, a tensor product discretization of the spacetime, with constant time steps $\Delta t$ and constant space steps $\Delta s$. However, one of the main features of this theorem is that it remains valid in the more general situation in which the spatial discretization is adapted at each time step, as illustrated in Figure 3. This property follows from the spacetime nature of the variational principle. Equations (64)-(67) and (78)-(81) have exactly the same form in this more general situation. The change in the spatial discretization is encoded in the dependence of the Lagrangian $\mathcal{L}_{d}$ on its first argument $\square_{a}^{j}$. This dependence enters in a nontrivial way in equations (65) and (79), through the partial derivatives of $\mathcal{L}_{d}$ with respect to the discrete time.

In the simpler case of a tensor product discretization, our algorithm can be a posteriori interpreted as arising from a spatial discretization of the problem, followed by a collision variational discretization in time, in the sense of Fetecau et al. [42]. However, we note that applying a priori such a discretization in two consecutive steps will, in general, neither preserve the multisymplectic character of the problem, nor the covariant Noether theorem at the discrete level, whereas a multisymplectic discretization, even in the case of a tensor product discretization, will allow for a simultaneous preservation of these two properties. In the general case of the discrete spacetime illustrated in Figure 3, which is potentially useful for a systematic development of adaptive meshes, such a discretization in two steps is not possible. 
Finally, as we already mentioned earlier, for simplicity of the exposition, we have assumed a single collision time, but our algorithm can equally well handle several collision times at different spatial nodes.

\section{Numerical tests}

5.1. Impact of an elastic beam on a rigid plate. We illustrate the discrete geometric framework of Section 4.2, with configuration space $\mathcal{C}^{a}$ (continuous and nonsmooth, see (6)), presenting the example of the elastic contact between a 3-dimensional geometrically exact beam model and a rigid plate.

Geometrically exact beam models are naturally formulated in terms of Lie group valued fields. Thus, it is important to retain the underlying Lie group and Lie algebra structure in the discretized model in order to recover the qualitative properties of the dynamics in the simulations (see Iserles et al. [67]). This has the added advantage of also minimizing numerical error.

Field theoretic description of beam dynamics. In geometrically exact beam models (Simó [123], Simó et al. [124]), the configuration of a beam is described by the line of centroids $\mathbf{r}$ and by a moving orthonormal basis

$$
\left\{\mathbf{d}_{1}(t, s), \mathbf{d}_{2}(t, s), \mathbf{d}_{3}(t, s)\right\}
$$

giving the orientation of the cross-section at each of its points. The attitude of this moving basis is described by a rotation matrix $\Lambda \in S O$ (3). Thus, the configuration space of the beam is the space of maps

$$
(\Lambda, \mathbf{r})=(\Lambda(t, s), \mathbf{r}(t, s)):[0, T] \times[0, L] \rightarrow S O(3) \times \mathbb{R}^{3}
$$

satisfying $\mathbf{d}_{I}(t, s)=\Lambda(t, s) \mathbf{E}_{I}, I=1,2,3,\left\{\mathbf{E}_{1}, \mathbf{E}_{2}, \mathbf{E}_{3}\right\}$ a fixed orthonormal basis. See Figure 4.

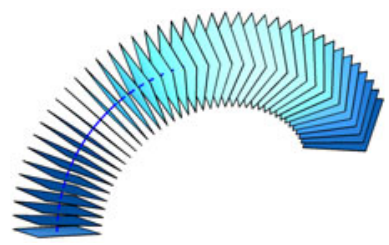

Figure 4. The geometrically exact model of the beam is defined by the position $\mathbf{r}(t, s) \in \mathbb{R}^{3}$ of the line of centroids and by the orientation $\Lambda(t, s) \in S O(3)$ of the cross-sections. This figure illustrates the discretized beam at a given time $t^{j}$ giving all $A$ cross-sections at the spatial points $s_{a}$. 
We work with the Lagrangian field theoretic description of geometrically exact beam models developed in Ellis et al. [34]. The parameter and the base spaces are, respectively, $U=[0,1] \times[0,1]$ and $X=\mathbb{R} \times \mathbb{R}$. We assume that

$$
U_{X}=\phi_{X}(U)=[0, T] \times[0, L] \subset X .
$$

The covariant configuration bundle is $\pi_{X Y}: Y=X \times S E(3) \rightarrow X$ and a field is $\varphi:[0, T] \times[0, L] \ni(t, s) \mapsto(t, s, g(t, s)) \in \mathbb{R}^{2} \times S E(3)$, where

$$
g(t, s):=(\Lambda(t, s), \mathbf{r}(t, s)) \in S E(3)
$$

are the configuration variables given in (82). The first jet extension of $\varphi$ has the expression $j^{1} \varphi(t, s)=\left(t, s, g(t, s), \partial_{t} g(t, s), \partial_{s} g(t, s)\right)$. The Lagrangian density $\mathcal{L}\left(t, s, g(t, s), \partial_{t} g(t, s), \partial_{s} g(t, s)\right)$ is easily described in terms of the convected angular and linear velocities and strains $\xi:=g^{-1} \partial_{t} g=:(\omega, \gamma)$ and $\eta:=g^{-1} \partial_{s} g=:(\Omega, \boldsymbol{\Gamma})$, namely,

$$
\begin{aligned}
\mathcal{L}\left(g, \partial_{t} g, \partial_{s} g\right) & =K(\xi)-\Phi(\eta)-\Pi(g) \\
& =\frac{1}{2}\langle\downarrow \rrbracket \xi, \xi\rangle-\frac{1}{2}\left\langle\mathbb{C}\left(\eta-\mathbf{E}_{6}\right),\left(\eta-\mathbf{E}_{6}\right)\right\rangle-\Pi(g) \\
& =: \overline{\mathcal{L}}(g, \xi, \eta),
\end{aligned}
$$

where $K(\xi), \Phi(\eta)$, and $\Pi(g)$ are, respectively, the kinetic energy density, the strain energy density, and the external (such as gravitational) potential energy density. In (83), $\xi, \eta$ are regarded as elements of $\mathbb{R}^{6}$; $\mathbb{J}$ is a $6 \times 6$ diagonal matrix whose diagonal elements are the principal moments of inertia and the mass of the cross-section; the linear strain tensor $\mathbb{C}$ is a $6 \times 6$ diagonal matrix, whose diagonal elements depend on the cross-sectional area, the principal moments of inertia of the cross-sections, Young's modulus, and Poisson's ratio;

$$
\mathbf{E}_{6}=(0,0,0,0,0,1) \in \mathbb{R}^{6} .
$$

Both $\mathbb{J}$ and $\mathbb{C}$ are assumed to be independent of $(t, s)$. The last equality of (83) defines the trivialized discrete Lagrangian $\overline{\mathcal{L}}$.

Recall from Demoures et al. [29] that the covariant Euler-Lagrange equations are given by

$$
\frac{d}{d t}(\mathbb{J} \xi)-\operatorname{ad}_{\xi}^{*}(\mathbb{\xi})=\frac{d}{d s} \frac{\partial \Phi}{\partial \eta}-\operatorname{ad}_{\eta}^{*} \frac{\partial \Phi}{\partial \eta}-g^{-1} \frac{\partial \Pi}{\partial g},
$$

where $\operatorname{ad}_{(\omega, \gamma)}^{*}(\boldsymbol{\mu}, \boldsymbol{v})=-(\boldsymbol{\omega} \times \boldsymbol{\mu}+\boldsymbol{\gamma} \times \boldsymbol{v}, \boldsymbol{\omega} \times \boldsymbol{v})$, with $\boldsymbol{\mu}, \boldsymbol{v} \in \mathfrak{s e}(3)^{*}$. We use the zero-traction boundary condition

$$
\frac{\partial \Phi}{\partial \eta}(t, 0)=\frac{\partial \Phi}{\partial \eta}(t, L)=0
$$


Consider the impact of the beam on a rigid plate. Assume that the thickness of the beam is small relative to its length. Thus, we can choose the impenetrability constraint to be independent of the rotation matrix $\Lambda(t, s)$. It is given by

$$
\psi: S E(3) \rightarrow \mathbb{R}^{+}, \quad \psi(\Lambda(t, s), \mathbf{r}(t, s))=\left\langle\mathbf{r}(t, s), \mathbf{E}_{3}\right\rangle \geqslant 0,
$$

where the rigid plate is the horizontal plane $z^{3}=0$ in $\mathbb{R}^{3}$. The constraint subdomain has thus the expression

$$
C:=\psi^{-1}\left(\left[0, \infty[)=S O(3) \times\left\{\left(z^{1}, z^{2}, z^{3}\right) \mid z^{3} \geqslant 0\right\}=: C_{1} \times C_{2} .\right.\right.
$$

Since the constraint depends solely on the position $\mathbf{r}=\left(r^{1}, r^{2}, r^{3}\right)$, the normal cone to $C$ at $(\Lambda, \mathbf{r})$ is

$$
N_{C}(\Lambda, \mathbf{r})=N_{C_{1}}(\Lambda) \times N_{C_{2}}(\mathbf{r}) \quad \text { with }\left\{\begin{array}{l}
N_{C_{1}}(\Lambda)=\{0\} \quad \text { for all } \Lambda \in S O(3), \\
N_{C_{2}}(\mathbf{r})= \begin{cases}]-\infty, 0] & \text { if } r^{3}=0, \\
\{0\} & \text { if } r^{3}>0, \\
\varnothing & \text { if } r^{3}<0,\end{cases}
\end{array}\right.
$$

where ] $-\infty, 0$ ] corresponds to the outward normal direction to $C_{2}$ at $\mathbf{r}$.

Multisymplectic variational integrator for the impact of a beam. For each rectangular base space $\square_{a}^{j} \in U_{d}^{\square}$, we define the triangles:

$$
\begin{aligned}
\triangle_{a}^{j} & =((j, a),(j+1, a),(j, a+1)), \\
\triangleright_{a}^{j+1} & =((j+1, a),(j, a),(j+1, a+1)), \\
\nabla_{a+1}^{j} & =((j, a+1),(j+1, a+1),(j, a)), \\
\triangleleft_{a+1}^{j+1} & =((j+1, a+1),(j, a+1),(j+1, a)) .
\end{aligned}
$$

Note that $\square_{a}^{j}=\triangle_{a}^{j} \cup \triangleleft_{a+1}^{j+1}=\nabla_{a+1}^{j} \cup \triangleright_{a}^{j+1}$; see Figure 5. The discrete trivialized Lagrangian $\mathcal{L}_{d}\left(\square_{a}^{j}, g_{a}^{j}, g_{a}^{j+1}, g_{a+1}^{j}, g_{a+1}^{j+1}\right)$ defined on $\square_{a}^{j}$ is then expressed as a sum
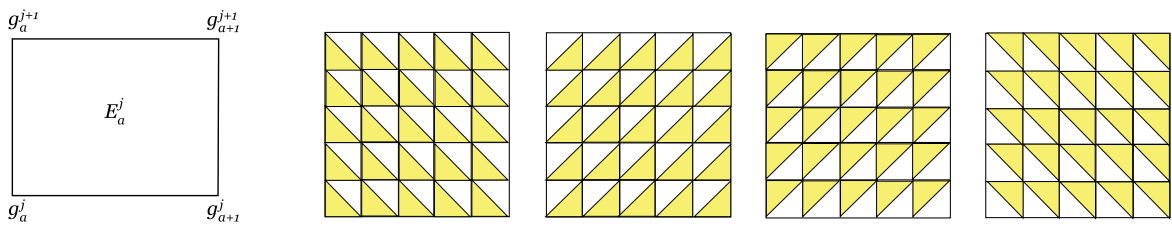

Figure 5. The rectangular base space $\square_{a}^{j}$ and the triangles $\triangle_{a}^{j}, \nabla_{a+1}^{j}, \triangleright_{a}^{j+1}, \triangleleft_{a+1}^{j+1}$. 
of discrete trivialized Lagrangians defined on triangular meshes. Fix $\Delta t$ and $\Delta s$. Take the discrete base-space configuration (53). Below we use the notation

$$
\Delta t^{j}:=\left\{\begin{array}{ll}
\Delta t & \text { if } j \notin\{i-1, \tilde{i}\} \\
\Delta t^{i-1}:=\tilde{t}-t^{i-1} & \text { if } j=i-1
\end{array} \text { and } \Delta \widetilde{t}:=t^{i}-\widetilde{t} .\right.
$$

Given a discrete first jet $j^{1} \varphi_{d}\left(\square_{a}^{j}\right)$, we define

$$
\begin{aligned}
& \xi_{a}^{j}:=\tau^{-1}\left(\left(g_{a}^{j}\right)^{-1} g_{a}^{j+1}\right) / \Delta t, \quad j \notin\{i-1, \widetilde{i}\}, \quad \xi_{a}^{i-1}:=\tau^{-1}\left(\left(g_{a}^{i-1}\right)^{-1} \widetilde{g}_{a}\right) / \Delta t^{i-1}, \\
& \widetilde{\xi}_{a}:=\tau^{-1}\left(\left(\widetilde{g}_{a}\right)^{-1} g_{a}^{i}\right) / \Delta \widetilde{t}, \quad \eta_{a}^{j}:=\tau^{-1}\left(\left(g_{a}^{j}\right)^{-1} g_{a+1}^{j}\right) / \Delta s, \quad j=0, \ldots, N,
\end{aligned}
$$

where $\tau: \mathfrak{s e}(3) \rightarrow S E(3)$ is a local diffeomorphism approximating the exponential map around the origin and satisfying $\tau(0)=\left(\mathbf{I}_{3}, 0\right)$.

The discrete Lagrangian $\overline{\mathcal{L}}_{d}: X_{d}^{\square} \times S E(3)^{4} \times \mathfrak{s e}(3)^{4} \rightarrow \mathbb{R}$ defined on a rectangle $\square_{a}^{j}$ is obtained by summing four discrete Lagrangians defined on the four triangular base spaces $\triangle_{a}^{j}, \triangleright_{a}^{j+1}, \nabla_{a+1}^{j}, \triangleleft_{a+1}^{j+1}$. This discrete Lagrangian reads

$$
\begin{aligned}
& \overline{\mathcal{L}}_{d}\left(\square_{a}^{j}, g_{a}^{j}, g_{a}^{j+1}, g_{a+1}^{j}, g_{a+1}^{j+1}\right)=\Delta s \Delta t^{j}\left[\frac{1}{4}\left\langle\mathbb{J} \xi_{a}^{j}, \xi_{a}^{j}\right\rangle+\frac{1}{4}\left\langle\mathbb{J} \xi_{a+1}^{j}, \xi_{a+1}^{j}\right\rangle\right. \\
& \left.\quad-\frac{1}{4}\left\langle\mathbb{C}\left(\eta_{a}^{j}-\mathbf{E}_{6}\right), \eta_{a}^{j}-\mathbf{E}_{6}\right\rangle-\frac{1}{4}\left\langle\mathbb{C}\left(\eta_{a}^{j+1}-\mathbf{E}_{6}\right), \eta_{a}^{j+1}-\mathbf{E}_{6}\right\rangle-\bar{\Pi}\left(g_{a}^{j}\right)\right],
\end{aligned}
$$

with $\bar{\Pi}\left(g_{a}^{j}, g_{a}^{j+1}, g_{a+1}^{j}, g_{a+1}^{j+1}\right):=\frac{1}{4}\left(\Pi\left(g_{a}^{j}\right)+\Pi\left(g_{a}^{j+1}\right)+\Pi\left(g_{a+1}^{j}\right)+\Pi\left(g_{a+1}^{j+1}\right)\right)$. This Lagrangian differs from the one in Demoures et al. [29] which is based only on triangles $\triangle_{a}^{j}$. The choice (87) greatly improves the behavior of the integrator, especially concerning the boundaries (see Demoures et al. [30]).

When there is contact, the discrete action functional $\mathfrak{S}_{d}^{n s}\left(\phi_{X_{d}}, \varphi_{d}\right)$ is of the form (55). The discrete impenetrability condition is given by (84) and the normal cone to the constraint subdomain has the expression (85). Thus, we can apply Theorem 5. We give below only the equations at contact time; see Demoures et al. [30] for the full discrete equations of motion.

The vertical discrete jump conditions are

$$
\begin{aligned}
& 2 \Delta s\left(-\widetilde{\mu}_{a}+\operatorname{Ad}_{\tau\left(\Delta t^{i-1} \xi_{a}^{i-1}\right)}^{*} \mu_{a}^{i-1}\right)+\Delta t\left(\widetilde{\lambda}_{a}-\operatorname{Ad}_{\tau\left(\Delta s \widetilde{\eta}_{a-1}\right)}^{*} \widetilde{\lambda}_{a-1}\right) \\
& =\Delta s \Delta t\left(\widetilde{g}_{a}\right)^{-1} D_{\widetilde{g}_{a}} \Pi\left(\widetilde{g}_{a}\right)+2 \widetilde{\lambda}_{a}\left(\widetilde{g}_{a}\right)^{-1} D_{g} \psi\left(\widetilde{g}_{a}\right),
\end{aligned}
$$

for all $a \in\{1, \ldots, A-1\}$, where $\widetilde{\lambda}_{a}$ is the Lagrange multiplier, where

$$
\mu_{a}^{j}:=\left(d \tau_{\Delta t^{j} \xi_{a}^{j}}^{-1}\right)^{*} \rrbracket \xi_{a}^{j}, \quad \lambda_{a}^{j}:=\left(d \tau_{\Delta s \eta_{a}^{j}}^{-1}\right)^{*} \mathbb{C}\left(\eta_{a}^{j}-\mathbf{E}_{6}\right) .
$$

Note that $\tilde{\lambda}_{a}=0$ unless the node $a$ is in contact, in which case we have $\tilde{\lambda}_{a}<0$. Note also that when the contact is on the boundaries $a \in\{0, A\}$, we have to modify 
the previous vertical condition by taking into account the discrete boundary conditions.

The horizontal discrete jump condition associated to the time component variations, giving the energy conservation during the impact:

$$
\sum_{a=0}^{A-1}\left(D_{h} \mathcal{L}_{a}^{i-1}-D_{h} \widetilde{\mathcal{L}}_{a}\right)=0 .
$$

If the contact is at one of the boundaries, we have

$$
\begin{aligned}
& \Delta s\left(-\widetilde{\mu}_{0}+\operatorname{Ad}_{\tau\left(\Delta t t^{i-1} \xi_{0}^{i-1}\right)}^{*} \mu_{0}^{i-1}\right)+\Delta t \widetilde{\lambda}_{0} \\
& \quad=\frac{1}{2} \Delta s \Delta t\left(\widetilde{g}_{0}\right)^{-1} D_{\widetilde{g}_{0}} \Pi\left(\widetilde{g}_{0}\right)+\widetilde{\lambda}_{0}\left(\widetilde{g}_{0}\right)^{-1} D_{g} \psi\left(\widetilde{g}_{0}\right),
\end{aligned}
$$

or

$$
\begin{aligned}
& \Delta s\left(-\tilde{\mu}_{A}+\operatorname{Ad}_{\tau(\Delta t i-1}^{* i-1} \xi_{A}^{i-1} \mu_{A}^{i-1}\right)-\Delta t \operatorname{Ad}_{\tau\left(\Delta s \widetilde{\eta}_{A-1}\right)}^{*} \widetilde{\lambda}_{A-1} \\
& \quad=\frac{1}{2} \Delta s \Delta t\left(\widetilde{g}_{A}\right)^{-1} D_{\widetilde{g}_{A}} \Pi\left(\widetilde{g}_{A}\right)+\widetilde{\lambda}_{A}\left(\widetilde{g}_{A}\right)^{-1} D_{g} \psi\left(\widetilde{g}_{A}\right) .
\end{aligned}
$$

Discrete momentum maps. The discrete Lagrangian $\mathcal{L}_{a}^{j}$ is invariant with respect to the action of the subgroup $H=S^{1} \times \mathbb{R}^{2}$ of $S E$ (3) on $S E$ (3), given by $\left(R_{z}, \mathbf{v}\right) \cdot(\Lambda, \mathbf{r})=\left(R_{z} \Lambda, \mathbf{v}+\mathbf{r}\right)$, where $R_{z}$ is rotation with axis $\mathbf{E}_{3}$, and $\mathbf{v}$ is a translation vector parallel to the plane $z^{3}=0$. As a consequence the Noether Theorem 6 holds. The four discrete momentum maps are computed using (50); see Demoures et al. [28, formula (32)] for similar formulas on triangles instead of squares.

Symplectic properties of the discrete time evolution. Associate to $\mathcal{L}_{d}$ a discrete Lagrangian

$$
\mathrm{L}_{d}\left(\mathbf{g}^{j}, \boldsymbol{\xi}^{j}\right):=\sum_{a=0}^{A-1} \mathcal{L}_{d}\left(\square_{a}^{j}, g_{a}^{j}, g_{a}^{j+1}, g_{a+1}^{j}, g_{a+1}^{j+1}\right)
$$

for the time evolution, $\mathbf{g}^{j}:=\left(g_{0}^{j}, \ldots, g_{A}^{j}\right), \xi^{j}:=\left(\xi_{0}^{j}, \ldots, \xi_{A}^{j}\right)$. If there is no impact, the discrete flow $\mathbf{g}^{j}$ is a symplectic integrator (see Demoures et al. [29]). This result and those in Fetecau et al. [42] imply that the discrete flow $\mathbf{g}^{j}$ defines a symplectic integrator in presence of impact. Analogously to results in Demoures et al. [29, Section 3.3], it is possible to construct the momentum maps associated to the time evolution. Thus, if the configuration is prescribed at the temporal extremities and zero-traction boundary conditions are imposed, then the discrete momentum map is conserved, even in the presence of contacts, supposing that the impenetrability constraints are invariant under the action of the subgroup $H$ of $S E(3)$. 
Numerical tests. We provide numerical tests of our integrator. We consider the initial value problem of a geometrically exact beam with length $L=0.5 \mathrm{~m}$ and square cross-section of side $a=0.05 \mathrm{~m}$, evolving with unconstrained extremities in space. The beam parameters are set to $\rho=10^{3} \mathrm{~kg} / \mathrm{m}^{3}, E=10^{3} \mathrm{~N} / \mathrm{m}^{2}$, $v=0.35$. The value of the gravitational acceleration is taken to be $g=10 \mathrm{~m} / \mathrm{s}^{2}$. Zero-traction boundary conditions are imposed at the two extremities of the beam. The initial conditions are given by the configuration $g_{a}^{0}$ and the initial speed $\xi_{a}^{0}$ at time $t=t^{0}$ for all positions $s_{0}, \ldots, s_{A}$. Taking the local diffeomorphism around the origin $\tau: \mathfrak{s e}(3) \rightarrow S E(3)$ to be the Cayley map given by (see Selig [121])

$$
\tau(\omega, \boldsymbol{\gamma}):=\left[\begin{array}{c}
\operatorname{cay} \omega \frac{4}{4+\|\boldsymbol{\omega}\|^{2}}\left(\mathbf{I}_{3}+\frac{1}{2} \omega+\frac{1}{4} \boldsymbol{\omega} \boldsymbol{\omega}^{T}\right) \boldsymbol{\gamma} \\
1
\end{array}\right],
$$

we choose

$$
\begin{aligned}
h_{0} & =\left(\left[\begin{array}{ccc}
1 & 0 & 0 \\
0 & \cos (0.5) & -\sin (0.5) \\
0 & \sin (0.5) & \cos (0.5)
\end{array}\right],\left[\begin{array}{l}
0 \\
0 \\
0
\end{array}\right]\right), \\
h_{a+1} & =h_{a} \tau\left(\Delta s \eta_{a}^{0}\right), \quad \forall a=0, \ldots, A-1,
\end{aligned}
$$

where $\eta_{a}^{0}=(1,4.5,1,0,0,1)$, for all $a=0, \ldots, A-1$, and

$$
\begin{aligned}
g_{0}^{1} & =\left(\left[\begin{array}{ccc}
1 & 0 & 0 \\
0 & \cos (0.5) & -\sin (0.5) \\
0 & \sin (0.5) & \cos (0.5)
\end{array}\right],\left[\begin{array}{c}
0 \\
-2 \cdot 10^{-5} \sin (0.5) \\
2 \cdot 10^{-5} \cos (0.5)
\end{array}\right]\right), \\
g_{a+1}^{1} & =g_{a}^{1} \tau\left(\Delta s \eta_{a}^{1}\right),
\end{aligned}
$$

for all $a=0, \ldots, A-1$, where $\eta_{a}^{1}=(1.004,4.522,0.996,-0.004,0,1)$. Hence $\xi_{a}^{0}=\frac{1}{\left(4 \cdot 10^{-5}\right)} \tau^{-1}\left(\left(h_{a}\right)^{-1} g_{a}^{1}\right), \quad$ and $\quad g_{a}^{0}=g_{a}^{1}\left(\tau\left(\Delta t \xi_{a}^{0}\right)\right)^{-1}, \quad \forall a=0, \ldots, A$.

We observe an excellent conservation of energy and of the discrete momentum map $\mathbf{J}_{\mathrm{L}_{d}}=\left(\mathbf{J}^{1}, \mathbf{J}^{2}, \mathbf{J}^{3}\right)$ associated with the $S^{1} \times \mathbb{R}^{2}$ symmetry (see Figure 6). We refer to Demoures et al. [30] for further numerical tests and discussions of the results. The configuration of the beam in contact, without friction, is illustrated in Figure 7.

5.2. Impact of elastic bars. We illustrate the theory of Section 4.3, with configuration space $\mathcal{C}^{c}$ (discontinuous with separation, see (8)), employing the standard benchmark example of elastic contacts between two bars which have been previously presented in Taylor and Papadopoulos [126]; see also Cirak and West [17] and Glocker [53]. 

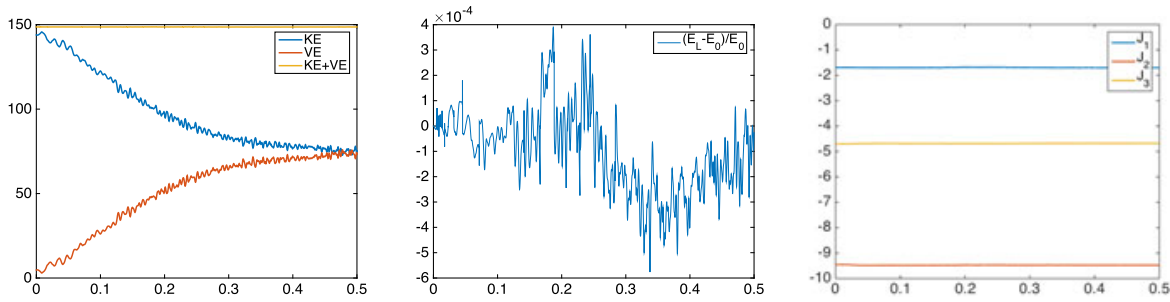

Figure 6. From left to right: total energy, relative error, and momentum maps. Beam composed of 20 elements with $\Delta t=0.00001 \mathrm{~s}$. Interval of time $[0,0.5]$.
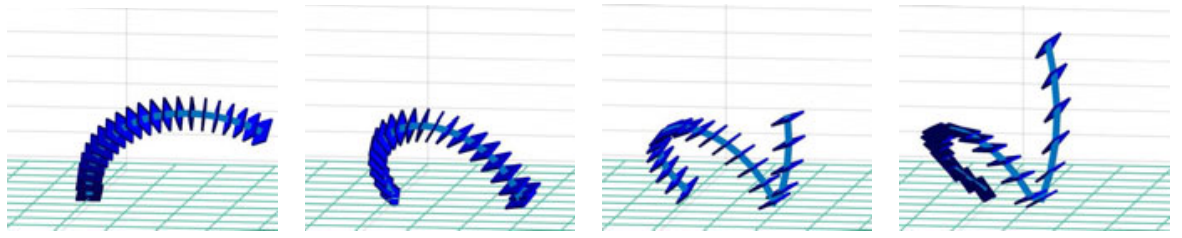

Figure 7. From left to right: configuration of the beam at times $t=0.0 \mathrm{~s}$, $t=0.0053 \mathrm{~s}, t=0.01 \mathrm{~s}, t=0.015 \mathrm{~s}$.

Field theoretic description. Let $L_{1}, L_{2}$ be the lengths of the two bars. The fields are $\varphi: X=[0, T] \times\left\{\left[0, L_{1}\right] \cup\left[0, L_{2}\right]\right\} \rightarrow M \times M=\mathbb{R} \times \mathbb{R}$. The configuration bundle is $\pi: Y=X \times \mathbb{R} \times \mathbb{R} \rightarrow X$, so the first jet bundle can be canonically identified with the vector bundle over

$$
Y=X \times \mathbb{R} \times \mathbb{R} \ni\left(t, s_{1}, s_{2}, \varphi_{1}\left(t, s_{1}\right), \varphi_{2}\left(t, s_{2}\right)\right),
$$

with fiber $\mathbb{R}^{2} \times \mathbb{R}^{2} \ni\left(\partial_{t} \varphi_{1}\left(t, s_{1}\right), \partial_{t} \varphi_{2}\left(t, s_{2}\right), \partial_{s_{1}} \varphi_{1}\left(t, s_{1}\right), \partial_{s_{2}} \varphi_{2}\left(t, s_{2}\right)\right)$. The Lagrangian density of each of the two 1-dimensional elastic bars labeled by $\alpha \in\{a, b\}$, is

$$
\begin{aligned}
& \mathcal{L}_{\alpha}\left(t, s_{\alpha}, \varphi\left(t, s_{\alpha}\right), \partial_{t} \varphi\left(t, s_{\alpha}\right), \partial_{s_{\alpha}} \varphi\left(t, s_{\alpha}\right)\right) \\
& \quad=\frac{1}{2}\left(m_{\alpha}\left|\partial_{t} \varphi\left(t, s_{\alpha}\right)\right|^{2}-\mathcal{E}_{\alpha} \mathcal{S}\left|\partial_{s_{\alpha}} \varphi\left(t, s_{\alpha}\right)-1\right|^{2}\right) d t \wedge d s_{\alpha} \\
& \quad=:\left(K_{\alpha}\left(\partial_{t} \varphi\left(t, s_{\alpha}\right)\right)-\Phi_{\alpha}\left(\partial_{s_{\alpha}} \varphi_{\alpha}\left(t, s_{\alpha}\right)\right)\right) d t \wedge d s_{\alpha},
\end{aligned}
$$

where $\mathcal{S}$ is area of the cross-section of the bar, $m_{\alpha}$ is the mass by unit length of the bar, and $\mathcal{E}_{\alpha}$ is the Young modulus. Applying Hamilton's principle to $\mathcal{L}_{a}+\mathcal{L}_{b}$ with given configurations at $t=0, T$, we get the wave equations

$$
m_{\alpha} \partial_{t t} \varphi_{\alpha}=\mathcal{E}_{\alpha} \mathcal{S} \partial_{s_{\alpha} s_{\alpha}} \varphi_{\alpha}, \quad \partial_{s_{\alpha}} \varphi_{\alpha}(t, 0)=1, \quad \partial_{s_{\alpha}} \varphi_{\alpha}\left(t, L_{\alpha}\right)=1, \quad \alpha \in\{a, b\} .
$$


Assume that the initial configuration and velocity of the beam are known. The two bars are moving against each other, subject to the impenetrability constraint

$$
\psi\left(t, s_{1}, s_{2}, \varphi_{1}, \varphi_{2}\right)=\varphi_{2}-\varphi_{1} \geqslant 0, \quad \forall t \in[0, T], \quad \forall s_{\alpha} \in\left[0, L_{\alpha}\right] .
$$

The corresponding constraint subdomain is defined by $C:=\psi^{-1}([0, \infty[)$.

Multisymplectic variational integrator for the impact of elastic bars. Following the definition (37) of the first jet extension $j^{1} \varphi_{d}$ of a discrete section $\varphi_{d}$ defined on $\phi_{X_{d}}\left(U_{d}^{\square}\right)$, we write $j^{1} \varphi_{d}\left(\square_{\alpha}^{j}\right)=\left(\square_{\alpha}^{j}, \varphi_{\alpha}^{j}, \varphi_{\alpha}^{j+1}, \varphi_{\alpha+1}^{j}, \varphi_{\alpha+1}^{j+1}\right)$. We approximate the Lagrangian (88) by the discrete Lagrangian $\mathcal{L}_{\alpha}^{j}:=\mathcal{L}_{\alpha, d}\left(j^{1} \varphi_{d}\left(\square_{\alpha}^{j}\right)\right)$, obtained by the generalized trapezoidal rule applied on the rectangles $\square_{\alpha}^{j}$, that is,

$$
\mathcal{L}_{\alpha}^{j}=\frac{1}{2} \Delta t \Delta s\left(K_{1}\left(v_{\alpha}^{j}\right)+K_{1}\left(v_{\alpha+1}^{j}\right)-\Phi_{1}\left(e_{\alpha}^{j}\right)-\Phi_{1}\left(e_{\alpha}^{j+1}\right)\right), \quad \alpha \in\{a, b\}
$$

$v_{\alpha}^{j}:=\left(\varphi_{\alpha}^{j+1}-\varphi_{\alpha}^{j}\right) / \Delta t^{j}$ is the discrete speed and $e_{\alpha}^{j}:=\left(\varphi_{\alpha+1}^{j}-\varphi_{\alpha}^{j}\right) / \Delta s$ the discrete strain. The discrete action functional $\mathfrak{S}_{d}^{n s}\left(\phi_{1 d}, \phi_{2 d}\right)$ is of the form (76). Given the discrete impenetrability condition

$$
\psi_{d}\left(\varphi_{a}^{j}, \varphi_{b}^{j}\right)=\varphi_{b}^{j}-\varphi_{a}^{j} \geqslant 0, \quad \forall a=0, \ldots, A, \quad \forall b=0, \ldots, B,
$$

and the impact time $\tilde{t}=\phi_{X_{d}}(\tilde{i})$, use Theorem 7 to get

(a) The vertical discrete jump conditions

$$
\left[\begin{array}{c}
\frac{\Delta s_{1}}{2} M_{1}\left(-\widetilde{v}_{A}+v_{A}^{i-1}\right)-\Delta t \mathcal{E}_{1} \mathcal{S}\left(\widetilde{e}_{A}-1\right) \\
\frac{\Delta s_{2}}{2} M_{2}\left(-\widetilde{v}_{0}+v_{0}^{i-1}\right)+\Delta t \mathcal{E}_{2} \mathcal{S}\left(\widetilde{e}_{0}-1\right)
\end{array}\right]=\lambda_{A, 0}\left[\begin{array}{c}
-1 \\
1
\end{array}\right] .
$$

(b) The horizontal discrete jump condition associated to the time component variations, giving the energy conservation during the impact:

$$
\sum_{a=0}^{A-1}\left(E_{a}^{i-1}-\widetilde{E}_{a}\right)+\sum_{b=0}^{B-1}\left(E_{b}^{i-1}-\widetilde{E}_{b}\right)=0 \quad \text { with } E_{a}^{j}=-D_{h} \mathcal{L}_{a}^{j}, \quad E_{b}^{j}=-D_{h} \mathcal{L}_{b}^{j} .
$$

See Demoures et al. [30] for the full discrete equations of motion outside of contact.

Discrete momentum maps. Since the discrete Lagrangian is spatially invariant, the corresponding Noether theorem holds for the discrete momentum maps. 

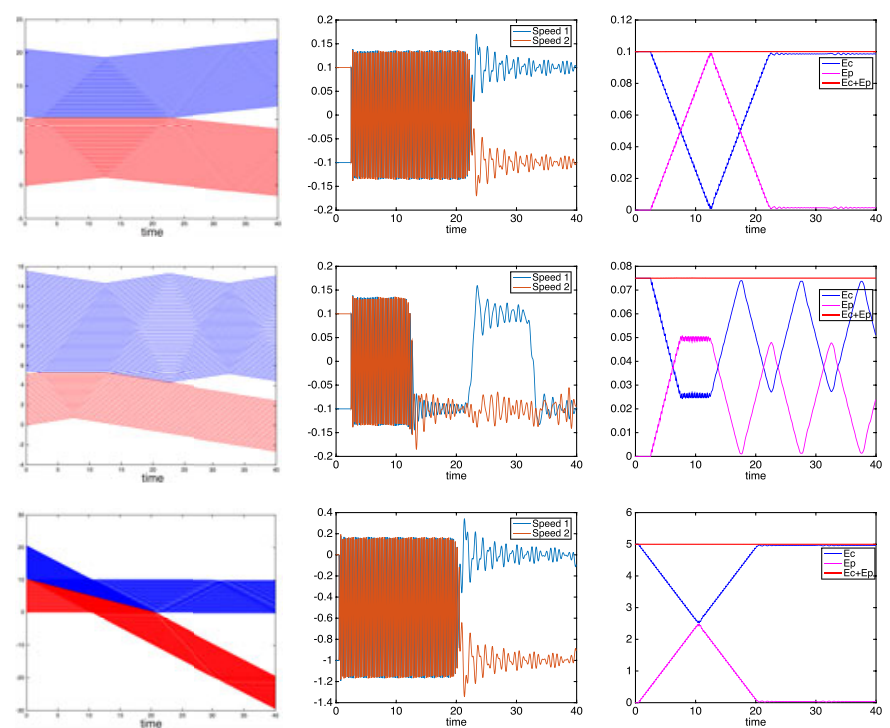
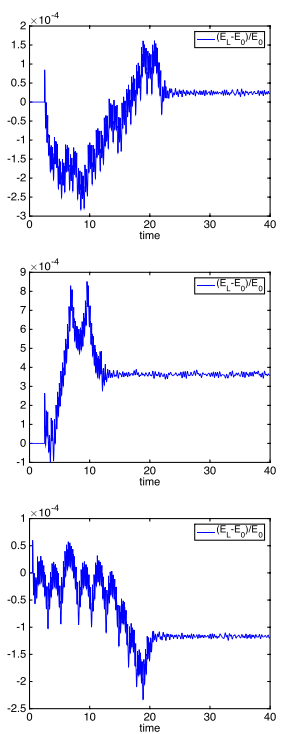

Figure 8. From left to right: displacement, speeds of the nodes in contact, total energy, and relative energy error. From top to bottom: (1) $L_{1}=L_{2}=10, M_{1}=$ $M_{2}=1, \mathcal{E}_{1}=\mathcal{E}_{2}=1, \ell_{1}=\ell_{2}=0.2, v_{1}=-0.1, v_{2}=0.1, \Delta t=0.015 \mathrm{~s}$, $T=40 \mathrm{~s}$; (2) $L_{1}=10, L_{2}=5, M_{1}=M_{2}=1, \mathcal{E}_{1}=\mathcal{E}_{2}=1, \ell_{1}=\ell_{2}=0.2$, $v_{1}=-0.1, v_{2}=0.1, \Delta t=0.015 \mathrm{~s}, T=40 \mathrm{~s}$; (3) $L_{1}=L_{2}=10, M_{1}=M_{2}=1$, $\mathcal{E}_{1}=\mathcal{E}_{2}=1, \ell_{1}=\ell_{2}=0.2, v_{1}=-1, v_{2}=0, \Delta t=0.015 \mathrm{~s}, T=40 \mathrm{~s}$.

Numerical tests. By the choice of the discrete Lagrangian (90), each bar is discretized as a mass-spring system with stiffness coefficients $\mathrm{k}_{\alpha}=\mathcal{E}_{\alpha} \mathcal{S} / \Delta s_{\alpha}$ $\mathrm{N} \mathrm{m}^{-1}$, and linear mass $m_{\alpha} \mathrm{kg} \mathrm{m}^{-1}$. The bars are $L_{\alpha}$ meters long and are discretized into $A$ and $B$ elements. The contact occurs at the extremities $a=A$ and $b=0$ of the bars (see Figure 2). Initial velocities $v_{\alpha} \mathrm{m} \mathrm{s}^{-1}$ are applied to the two bars.

In Figure 8 below, we present the result of our algorithm for the longitudinal impact of identical and nonidentical bars with various values for the lengths, densities, and initial velocities. In each case, we observe that the energy is conserved during and after the impact within $10^{-5}$. Consistently with the theoretical prediction, the momentum map, associated with the invariance relative to translations, is perfectly conserved. We refer to Demoures et al. [30] for further numerical tests and discussions of the results.

During the persistent contact phase, we observe rapid velocity oscillations as the masses bounce rapidly against each other, especially for two identical bars. While the averaged velocity is correct, these fine-scale oscillations can be 
interpreted as spurious, as they do not properly capture the physical behavior. In Demoures et al. [30], we propose a slight modification of the discrete Lagrangian at the extremities of the bar. This then gives rise to an important modification of the algorithm presented here that does not even use Lagrange multipliers and eliminates these spurious oscillations.

Conclusions. We develop the theory of multisymplectic variational integrators for nonsmooth continuum mechanics with constraints by combining, at the continuous and discrete levels, the variational multisymplectic formulation of nonsmooth continuum mechanics with the generalized Lagrange multiplier approach for optimization problems with nonsmooth constraints.

The resulting integrators verify a spacetime multisymplectic formula, generalizing the well-known symplectic property of time integrators, and exhibit the excellent energy conservation property, common to symplectic integrators. In the presence of symmetry, a discrete version of the Noether theorem is verified. Once a discretization of spacetime and the Lagrangian are implemented, the derivation of the integrator follows uniquely by applying a variational principle or, more generally, the generalized Lagrange multiplier approach. In this sense, our approach is systematic, general, and does not depend on the specific problem under consideration. The numerical scheme and the validity of the discrete conservation laws are illustrated with the help of two basic examples, namely, the impact of an elastic body on a rigid plate and the collision of two elastic 1-dimensional bars.

\section{Acknowledgments}

We thank A. Curnier for many helpful discussions and are grateful to him for allowing us to quote in the introduction certain aspects of the historical background presented in his papers to which we added the original references. We thank the three anonymous referees for their excellent suggestions on how to improve the paper. F.D. was partially supported by ERC Advanced grant 267382 FCCA. F.G.B. was partially supported by ANR project GEOMFLUID (ANR14-CE23-0002-01). T.S.R. was partially supported by Swiss NSF grant NCCR SwissMAP.

\section{References}

[1] P. Alart, 'Multiplicateurs augmentés et méthode de Newton généralisée pour contact avec frottement', Report, Document LMA-DME-EPFL, Lausanne (1988).

[2] P. Alart and A. Curnier, 'A mixed formulation for frictional contact problems prone to Newton like solution methods', Comput. Methods Appl. Mech. Engrg. 92(3) (1991), $353-375$. 
[3] F. Armero and E. Petocz, 'Formulation and analysis of conserving algorithms for frictionless dynamic contact/impact problems', Comput. Methods Appl. Mech. Engrg. 158 (1998), 269-300.

[4] K. J. Arrow and R. M. Solow, 'Gradient methods for constrained maxima with weakened assumptions', in Studies in Linear and Nonlinear Progrmming (eds. K. J. Arrow, L. Hurwitz and H. Uzawa) (Stanford University Press, Stanford, 1958), 166-176.

[5] H. Attouch and R. Wets, 'Approximation and convergence in nonlinear optimization', in Nonlinear Programming 4 (eds. O. Mangasarian, R. Meyer and S. Robinson) (Academic Press, New York-London, 1981), 367-394.

[6] H. Attouch and R. Wets, 'A convergence theory for saddle functions', Trans. Amer. Math. Soc. 280 (1983), 1-41.

[7] H. Attouch and R. Wets, 'Epigraphical analysis', in Analyse Non Linéaire (eds. H. Attouch, J.-P. Aubin, F. Clarke and I. Ekeland) (Gauthier-Villars, Paris, 1989), 73-100.

[8] E. J. Beltrami, 'On infinite-dimensional convex programs', J. Comput. System Sci. 1(4) (1967), 323-329.

[9] D. P. Bertsekas, 'On penalty and multiplier methods for constrained minimization', in Nonlinear Programming 2 (eds. O. L. Mangasarian, R. Meyer and S. Robinson) (Academic Press, New York, 1975), 165-191.

[10] D. P. Bertsekas, 'Necessary and sufficient conditions for a penalty method to be exact', Math. Program. 9 (1975), 87-99.

[11] D. P. Bertsekas, 'Combined primal-dual and penalty methods for constrained minimization', SIAM J. Control 13(3) (1975), 521-544.

[12] D. P. Bertsekas, 'On the method of multipliers for convex programming', IEEE Trans. Automat. Control (1975), 385-388.

[13] G. Bouligand, Précis de mécanique rationnelle à l'usage des élèves des facultés des sciences (Libraire Vuibert, Paris, 1925).

[14] T. Bridges and S. Reich, 'Multi-symplectic integrators: numerical schemes for Hamiltonian PDEs that conserve symplecticity', Phys. Lett. A 284(4-5) (2001), 184-193.

[15] J. D. Buys, 'Dual algorithms for constrained optimization problems', PhD Thesis, University of Leiden, 1972.

[16] L. Carnot, Essai sur les machines en général (Dijon, Defay, 1783).

[17] F. Cirak and M. West, 'Decomposition contact response (DCR) for explicit finite element dynamics', Internat. J. Numer. Methods Engrg. 64 (2005), 1078-1110.

[18] F. H. Clarke, Optimization and Nonsmooth Analysis, Classics in Applied Mathematics, 5 (SIAM, Philadelphia, PA, 1990).

[19] R. Clausius, 'On the mean length of the paths described by the separate molecules of gaseous bodies on the occurrence of molecular motion: together with some other remarks upon the mechanical theory of heat', Phil. Mag. 4(17) (1859), 81-91.

[20] R. Courant, 'Über direkte Methoden in der Variationsrechnung und über Verwandte Fragen', Math. Ann. 97 (1927), 711-736.

[21] R. Courant, 'Variational methods for the solution of problems of equilibrium and vibration', Bull. Amer. Soc. 49 (1943), 1-23.

[22] R. Courant, Methods of Mathematical Physics, Vol. 2 (Wiley-Interscience, New York, 1962).

[23] A. A. Cournot, 'Sur les percussions entre deux corps durs, qui se choquent en plusieurs points', Bull. Sci. Math., Astron., Phys. Chim. 7 (1827), 4-11.

[24] A. A. Cournot, 'Extension du principe des vitesses virtuelles au cas où les conditions de liaison du système sont exprimées par des inégalités', Bull. Sci. Math., Astron., Phys. Chim. 8 (1827), 165-170. 
[25] A. Curnier, 'Unilateral contact, mechanical modeling', in New Developments in Contact Problems (eds. P. Wriggers and P. Panagiotopoulos) CISM Courses and Lectures, 388 (International Center for Mechanical Sciences, Springer, Wien, 1999), 1-54.

[26] A. Curnier, Contact Mechanics and Nonsmooth Tribology, (EPFL, 2011), in preparation.

[27] E. Delassus, 'Les diverses formes du principe de d'Alembert et les équations générales du mouvement des systémes soumis à des liaisons d'ordre quelconques', Comptes Rendus T. CLVI (1913), 205-209.

[28] F. Demoures, F. Gay-Balmaz, M. Kobilarov and T. S. Ratiu, 'Multisymplectic Lie algebra variational integrator for a geometrically exact beam in $\mathbb{R}^{3}$, Commun. Nonlinear Sci. Numer. Simul. 19 (2014), 3492-3512.

[29] F. Demoures, F. Gay-Balmaz and T. S. Ratiu, 'Multisymplectic variational integrator and space/time symplecticity', Anal. Appl. 14(3) (2016), 341-391.

[30] F. Demoures, F. Gay-Balmaz, M. Desbrun, T. S. Ratiu and A. Aragon, 'A multisymplectic integrator for elastodynamic frictionless impact problems', Preprint, 2015.

[31] A. L. Dontchev and R. T. Rockafellar, Implicit Functions and Solution mappings. A View from Variational Analysis, 2nd edn, Springer Series in Operations Research and Financial Engineering (Springer, New York, 2014).

[32] P. Duhem, Hydrodynamique, élasticité et acoustique (A. Hemann, Paris, 1891).

[33] I. Ekeland and R. Temam, Analyse convexe et problèmes variationnels, 2nd edn, Convex Analysis and Variational Problems (Dunod-Gauthier-Villars, 1974), 1976, Classics in Applied Mathematics, 28, SIAM, Philadelphia, PA, 1999.

[34] D. Ellis, F. Gay-Balmaz, D.D. Holm, V. Putkaradze and T. S. Ratiu, 'Symmetry reduced dynamics of charged molecular strands', Arch. Rat. Mech. Anal. 197(2) (2010), 811-902.

[35] L. Euler, 'Mechanica sive motus scientia analytice exposita', Opera Omnia II(1) (1736).

[36] L. Euler, 'Methodus inveniendi lineas curvas', Opera Omnia I(24) (1744).

[37] L. Euler, 'Découverte d'un nouveau principe de mécanique (1750)', Mémoires l'Acad. Sci. Berlin 6 (1752), 185-217.

[38] J. Farkas, 'Die algebraische Grundlage der Anwendungen des machanischen Pricips von Fourier', Math. Naturwiss. Ber. Ungarn 16 (1899), 154-157.

[39] J. Farkas, 'Theorie der einfachen Ungleichungen', J. Reine Angew Math. 124 (1901), 1-27.

[40] W. Fenchel, Convex Cones, Sets and Functions, Lecture Notes (Princeton University, Princeton, NJ, 1951).

[41] R. C. Fetecau, J. E. Marsden and M. West, 'Variational multisymplectic formulations of nonsmooth continuum mechanics', in Perspectives and Problems in Nonlinear Science (Springer, New York, 2003), 229-261.

[42] R. C. Fetecau, J. E. Marsden, M. Ortiz and M. West, 'Nonsmooth Lagrangian mechanics and variational collision integrators', SIAM J. Appl. Dyn. Syst. 2 (2003), 381-416.

[43] A. V. Fiacco and G. P. McCormick, Nonlinear Programming, Classics in Applied Mathematics, 4 (SIAM, Philadelphia, PA, 1990), Reprint of the 1968 original.

[44] A. F. Filippov, 'Differential equations with discontinuous right-hand side (Russian)', Mat. Sb. (N.S.) 51(93) (1960), 99-128. AMS Transl., Series 2, 42, 1964, pp. 199-231.

[45] A. F. Filippov, 'Classical solutions of differential equations with multivalued right-hand side', SIAM J. Control 5 (1967), 609-621.

[46] A. F. Filippov, Differential Equations with Discontinuous Right-Hand Sides (Kluwer Academic, Norwell, MA, 1988).

[47] A. Forsgren, P. E. Gill and M. H. Wright, 'Interior methods for nonlinear optimization', SIAM Rev. 44(4) (2002), 525-597. 
[48] M. Fortin and R. Glowinski, Méthodes de lagrangien augmenté. Applications la résolution numérique de problèmes aux limites (Dunod, Paris, 1982).

[49] J. Fourier, 'Mémoire sur la statique contenant la démonstration du principe des vitesses virtuelles et la théorie des moments', J. Ec. Polytech. (1798), V ${ }^{e}$ Cahier, 20-64. Euvres de Fourier, tome second, 477-521, Gauthier-Villars et Fils, Paris, 1840.

[50] J. Fourier, 'Solution d'une question particulière du calcul des inégalites', Nouv. Bull. Sci. Soc. Philomatique Paris. Euvres de Fourier (1826), Gauthier-Villars et Fils, 1840.

[51] C. F. Gauß, 'Über ein neues allgemeines Grundgesetz der Mechanik', J. Reine Angew. Math. 4 (1829), 232-235.

[52] C. Glocker, Set-Valued Force Laws Dynamics of Non-smooth Systems, Lecture Notes in Applied Mechanics, 1 (Springer, Berlin, 2001).

[53] C. Glocker, 'Concepts for modeling impacts without friction', Acta Mech. 168 (2004), 1-19.

[54] R. Glowinski and A. Marocco, 'On the solution of a class of non-linear Dirichlet problems by penalty-duality method and finite elements of order one', C. R. Acad. Sci. Paris 278A (1974), 1649-1652.

[55] R. Glowinski and P. Le Tallec, Augmented Lagrangian and Operator Splitting Methods in Nonlinear Mechanics (SIAM, Philadelphia, PA, 1989).

[56] R. Glowinski, J. L. Lions and R. Tremolières, Analyse numérique des inéquations variationnelles, Tome 1 et 2, Méth. Math. Inf., 5 (Dunod, Paris, 1976).

[57] M. J. Gotay, J. Isenberg and J. E. Marsden, Momentum Maps and Classical Fields (Caltech, 2006).

[58] P. C. Haarhoff and J. D. Buys, 'A new method for the optimization of a nonlinear function subject to nonlinear constraints', Comput. J. 13 (1970), 178-184.

[59] E. Hairer, C. Lubich and G. Wanner, Geometric Numerical Integration, Structure-Preserving Algorithms for Ordinary Differential Equations, Springer Series in Computational Mathematics, 31 (Springer, Heidelberg, 2010).

[60] G. Hamel, 'Die Axiome der Mechanik', in Handbuch der Physik (eds. H . Geiger and K. Scheel) Band 5, Grundlagen der Mechanik, Mechanik der Punkte und Starren Körper (Springer, Berlin, 1927).

[61] G. Hamel, Theoretische Mechanik Eine einheitliche Einführung in die gesamte Mechanik, Grundlehren der Mathematischen Wissenschaften, 57 (Springer, Berlin-GöttingenHeidelberg, 1949), Corrected reprint of the 1949 edition, Springer, Berlin-New York, 1978.

[62] Q. C. He, J. J. Telega and A. Curnier, 'Unilateral contact of two solids subject to large deformations: formulation and existence results', Math. Phys. Eng. Sci. 452(1955) (1996), 2691-2717.

[63] J. H. Hefgaard and A. Curnier, 'An augmented Lagrangian method for discrete large-slip contact problems', Internat. J. Numer. Methods Engrg. 36 (1993), 569-593.

[64] H. Hertz, 'Über die Berührung fester elastischer Körper', J. Reine Angew. Math. 92 (1881), $156-171$.

[65] M. R. Hestenes, 'Multiplier and gradient methods', J. Optim. Theory Appl. 4(5) (1969), 303-320.

[66] T. J. R. Hughes, R. L. Taylor, J. L. Sackman, A. Curnier and W. Kanoknukulchai, 'A finite element method for a class of contact-impact problems', Comput. Methods Appl. Mech. Engrg. 8 (1975), 249-276.

[67] A. Iserles, H. Z. Munthe-Kaas, S. P. Nørsett and A. Zanna, 'Lie-group methods', Acta Numer. 9 (2000), 215-365.

[68] K. Ito and K. Kunisch, 'An augmented Lagrangian technique for variational inequalities', Appl. Math. Optim. 21(3) (1990), 223-241. 
[69] G. Johnson, S. Leyendecker and M. Ortiz, 'Discontinuous variational time integrators for complex multibody collisions', Internat. J. Numer. Methods Engrg. 100 (2014), 871-913.

[70] C. Kane, E. A. Repetto, M. Ortiz and J. E. Marsden, 'Finite element analysis of nonsmooth contact', Comput. Methods Appl. Mech. Engrg. 180 (1999), 1-26.

[71] W. Karush, 'Minima of functions of several variables with inequalities as side constraints', M.Sc. Thesis, Department of Mathematics, University of Chicago, USA, 1939.

[72] A. Klarbring, 'A mathematical programming approach to three-dimensional contact problems with friction', Comput. Methods Appl. Mech. Engrg. 58(2) (1986), 175-200.

[73] H. W. Kuhn and A. W. Tucker, 'Nonlinear Programming', in Proceedings of 2nd Berkeley Symposium (University of California Press, Berkeley, 1951), 481-492.

[74] J. L. de Lagrange, Méchanique Analitique, chez la Veuve Desaint, Libraire, rue du Foin S. Jacques, Paris, 1788; reprinted by Éditions Jacques Gabay, Paris, 1989.

[75] J. L. de Lagrange, Théorie des Fonctions Analytiques (Imprim. Rép., Paris, 1797).

[76] T. A. Laursen, Computational Contact and Impact Mechanics (Springer, Berlin, 2002).

[77] A. M. Legendre, Nouvelles Méthodes Pour la d'Étermination des Orbites de Comètes (Courcier, Paris, 1806).

[78] R. I. Leine and C. Glocker, 'A set-valued force law for spatial Coulomb-Contensou friction', Eur. J. Mech. A Solids 22(2) (2003), 193-216.

[79] R. I. Leine and H. Nijmeijer, Dynamics and Bifurcations of Non-smooth Mechanical Systems, Lecture Notes in Applied and Computational Mechanics, 18 (Springer, Berlin, 2004).

[80] A. Lew, J. E. Marsden, M. Ortiz and M. West, 'Asynchronous variational integrators', Arch. Ration. Mech. Anal. 167(2) (2003), 85-146.

[81] A. Lew, J. E. Marsden, M. Ortiz and M. West, 'Variational time integrators', Internat. J. Numer. Methods Engrg. 60(1) (2004), 153-212.

[82] J. E. Marsden, G. W. Patrick and S. Shkoller, 'Mulltisymplectic geometry, variational integrators and nonlinear PDEs', Comm. Math. Phys. 199 (1998), 351-395.

[83] J. E. Marsden and M. West, 'Discrete mechanics and variational integrators', Acta Numer. 10 (2001), 357-514.

[84] B. Martinet, 'Regularisation d'inéquations variationnelles par approximations successives', ESAIM Math. Model. Numer. Anal. 4(R3) (1970), 154-158.

[85] J.-J. Moreau, 'Fonctions convexes duales et points proximaux dans un espace hilbertien', C. R. Acad. Sci. Paris 255 (1962), 2897-2899.

[86] J.-J. Moreau, 'Les liaisons unilatérales et le principe de Gauss', C. R. Acad. Sci. Paris 256 (1963), 871-874.

[87] J.-J. Moreau, 'Théorèmes “inf-sup,”, C. R. Acad. Sci. Paris 258 (1964), 2720-2722.

[88] J.-J. Moreau, 'Proximité et dualité dans un espace hilbertien', Bull. Soc. Math. France 93 (1965), 273-299.

[89] J.-J. Moreau, Fonctionnelles Convexes, Sém EDP (Collège de France, Paris, 1966).

[90] J.-J. Moreau, 'La notion de sur-potentiel et les liaisons unilatérales en élastostatique', C. R. Acad. Sci. Paris Ser. A-B 267 (1968), A954-A957.

[91] J.-J. Moreau, 'Systèmes élastoplastiques de liberté finie', Travaux du Séminaire d'Analyse Convexe 3(12) (1973), 33 pp. Secrétariat des Math., Publ. No. 125. U.E.R. Math., Univ. Sci. Tech. Languedoc, Montpellier, 1973.

[92] J.-J. Moreau, On Unilateral Constraints, Friction and Plasticity, Centro Internaz. Mat. Estivo (C.I.M.E.), II Ciclo Bressanone, 1973, 171-322, Edizioni Cremonese, Roma, 1974.

[93] J.-J. Moreau, 'Liaisons unilatérales sans frottement et chocs inélastiques', C. R. Acad. Sci. Paris Ser. II Méc. Phys. Chim. Sci. Univers Sci. Terre 296(19) (1983), 1473-1476. 
[94] J.-J. Moreau, 'Une formulation du contact à frottement sec; application au calcul numérique', C. R. Acad. Sci. Paris Sér. II 302 (1986), 799-801.

[95] J.-J. Moreau, 'Unilateral contact and dry friction in finite freedom dynamics', in Nonsmooth Mechanics and Applications, (eds. J.-J. Moreau and P. D. Panagiotopoulos) CISM Courses and Lectures, 302 (International Center for Mechanical Sciences, Springer, 1988), 1-82.

[96] J.-J. Moreau, 'Jump functions of a real interval to a Banach space', Ann. Fac. Sci. Toulouse Math. (6) 5(suppl.) (1989), 77-91.

[97] J.-J. Moreau, 'Contact et frottement en dynamique des systèmes de corps rigides', Revue Européenne des Éléments Finis 9 (2000), 9-28.

[98] J.-J. Moreau and P. D. Panagiotopoulos, Non-Smooth Mechanics and Applications, 2nd edn, CISM Courses and Lectures, 302 (International Center for Mechanical Sciences, Springer, Wien, New York, 1988).

[99] M. V. Ostrogradsky, 'Considérations générales sur les momen(t)s des forces', Mém. Acad. Imp. Sci. Saint-Petersbourg, Sixième Série, Sciences Math. et Phys., 1835-1838 1 (1834), 129-150. Submitted November 7, 1834.

[100] M. V. Ostrogradsky, 'Mémoire sur les déplacemen(t)s instantanés des systèmes assujettis à des conditions variables', Mém. Acad. Imp. Sci. St. Petersbourg, Sixième Série, Sciences Math. et Phys., 1835-1838 1 (1838), 565-600. Submitted April 20, 1938.

[101] P. Painlevé, 'Sur les lois du frottement de glissemment', C. R. Acad. Sci. Paris 121 (1895), $112-115$.

[102] A. Pandolfi, C. Kane, J. E. Marsden and M. Ortiz, 'Time-discretized variational formulation of non-smooth frictional contact', Internat. J. Numer. Methods Engrg. 53 (2002), 1801-1829.

[103] P. Papadopoulos, 'A nonlinear programming approach to the unilateral contact and frictionboundary value problem in the theory of elasticity', Ing. Arch. 44 (1975), 421-432.

[104] P. Papadopoulos and R. L. Taylor, 'A mixed formulation for the finite element solution of contact problems', Comput. Methods Appl. Mech. Engrg. 94 (1992), 373-389.

[105] M. J. D. Powell, 'A method for nonlinear constraints in minimization problems', in 1969 Optimization (Sympos., Univ. Keele, Keele, 1968) (ed. R. Fletcher) (Academic Press, New York, NY, 1969), 283-298.

[106] G. Pietrzak, 'Continuum mechanics modelling and augmented Lagrangian formulation of large deformation frictional contact problems', PhD Thesis, EPFL, 1997.

[107] A. Prékopa, 'On the development of optimization theory', Amer. Math. Monthly 87(7) (1980), 527-542.

[108] R. T. Rockafellar, 'Convex functions and dual extremum problems', PhD Thesis, Harvard University, Cambridge, MA, 1963.

[109] R. T. Rockafellar, 'Characterization of the subdifferentials of convex functions', Pacific J. Math. 17 (1966), 497-510.

[110] R. T. Rockafellar, 'Convex functions, monotone operators and variational inequalities', in Theory and Applications of Monotone Operators (ed. A. Ghizzetti) (Tipografia Oderisi Editrice, Gubbio, Italy, 1968), 34-65.

[111] R. T. Rockafellar, Convex Analysis (Princeton, NJ, 1970).

[112] R. T. Rockafellar, 'Integrals which are convex functionals. II', Pacific J. Math. 39 (1971), 439-469.

[113] R. T. Rockafellar, 'Penalty methods and augmented Lagrangians in nonlinear programming', in Fifth Conference on Optimization Techniques (eds. R. Conti and A. Ruberti) (Springer, Berlin, 1973), 518-525. 
[114] R. T. Rockafellar, 'Augmented Lagrange multiplier functions and duality in nonconvex programming', SIAM J. Control 12 (1974), 268-285.

[115] R. T. Rockafellar, 'Monotone operators and the proximal point algorithm', SIAM J. Control Optim. 14 (1976a), 877-898.

[116] R. T. Rockafellar, 'Augmented Lagrangians and applications of the proximal point algorithm in convex programming', Math. Oper. Res. 1 (1976b), 97-116.

[117] R. T. Rockafellar, 'Monotone operators and augmented Lagrangian methods in nonlinear programming', in Nonlinear Programming 3 (ed. O. L. Mangasarian et al.) (Academic Press, New York-London, 1978), 1-25.

[118] R. T. Rockafellar, 'Proximal subgradients, marginal values, and augmented Lagrangians in nonconvex optimization', Math. Oper. Res. 6 (1981), 427-437.

[119] R. T. Rockafellar, 'Lagrange multipliers and optimality', SIAM Rev. 35(2) (1993), 183-238.

[120] R. T. Rockafellar and R. J.-B. Wets, Variational Analysis, Grundlehren der Mathematischen Wissenschaften, 317 (Springer, Berlin, 1998).

[121] J. N. Selig, 'Cayley maps for SE(3)', in Proceedings of the 12th IFToMM World Congress, Besançon, June 18-21, 2007 (eds. J.-P. Merlet and M. Dahan) (2007), paper A270.

[122] S. Signorini, 'Sopra alcune questioni di elastostatica', Atti della Societa Italiana per il Progresso delle Scienze (1933).

[123] J. C. Simó, 'A finite strain beam formulation. The three-dimensional dynamic problem. Part I', Comput. Methods Appl. Mech. Engrg. 49(1) (1985), 55-70.

[124] J. C. Simó, J. E. Marsden and P. S. Krishnaprasad, 'The Hamiltonian structure of nonlinear elasticity: the material and convective representations of solids, rods, and plates', Arch. Ration. Mech. Anal. 104(2) (1988), 125-183.

[125] C. Studer, R. I. Leine and C. Glocker, 'Step size adjustment and extrapolation for timestepping schemes in non-smooth dynamics', Internat. J. Numer. Methods Engrg. 76 (2008), 1747-1781.

[126] R. L. Taylor and P. Papadopoulos, 'On a finite element method for dynamic contact/impact problems', Internat. J. Numer. Methods Engrg. 36 (1993), 2123-2140.

[127] W. Thomson and P. G. Tait, Treatise on Natural Philosophy, Vol. 1 (Oxford University Press, Oxford, 1867).

[128] J. Vankerschaver, C. Liao and M. Leok, 'Generating functionals and Lagrangian partial differential equations', J. Math. Phys. 54(8) (2013), 082901, 22 pp.

[129] A. P. Wierzbicki, 'A penalty function shifting method in constrained static optimization and its convergence properties', Archiw. Autom. Telemech. 16 (1971), 395-416.

[130] P. Wriggers, Computational Contact Mechanics, 2nd edn (Springer, Berlin, Heidelberg, 2006).

[131] P. Wriggers and T. A. Laursen (Eds.), Computational Contact Mechanics, CISM Courses and Lectures, 498 (Springer, Wien-New York, 2007).

[132] K. Yosida, Functional Analysis (Springer, New York, 1965). 